\title{
Evaluation of phase chemistry and petrochemical aspects of Samchampi-Samteran differentiated alkaline complex of Mikir Hills, northeastern India
}

\author{
Abhishek Saha ${ }^{1, *}$, Sohini Ganguly ${ }^{1}$, Jyotisankar Ray ${ }^{1}$ and Nilanjan Chaterjee ${ }^{2}$ \\ ${ }^{1}$ Department of Geology, University of Calcutta, 35, Ballygunge Circular Road, Kolkata 700 019, India. \\ ${ }^{2}$ Department of Earth, Atmospheric and Planetary Sciences, Massachusetts Institute of Technology, \\ Cambridge, Massachusetts 02139, U.S.A. \\ *e-mail: asaha.geocal@gmail.com
}

The Samchampi-Samteran alkaline complex occurs as a plug-like pluton within the Precambrian granite gneisses of Mikir Hills, Assam, northeastern India and it is genetically related to Sylhet Traps. The intrusive complex is marked by dominant development of syenite within which ijolitemelteigite suite of rocks is emplaced with an arcuate outcrop pattern. Inliers of alkali pyroxenite and alkali gabbro occur within this ijolite-melteigite suite of rocks. The pluton is also traversed by younger intrusives of nepheline syenite and carbonatite. Development of sporadic, lumpy magnetite ore bodies is also recorded within the pluton. Petrographic details of the constituent lithomembers of the pluton have been presented following standard nomenclatorial rules. Overall pyroxene compositions range from diopside to aegirine augite while alkali feldspars are typically orthoclase and plagioclase in syenite corresponds to oligoclase species. Phase chemistry of nepheline is suggestive of Na-rich alkaline character of the complex. Biotite compositions are typically restricted to a uniform compositional range and they belong to 'biotite' field in the relevant classification scheme. Garnets (developed in syenite and melteigite) typically tend to be Ti-rich andradite, which on a closer scan can be further designated as melanites. Opaque minerals mostly correspond to magnetite. Use of Lindsley's pyroxene thermometric method suggests an equilibration temperature from $\sim 450^{\circ}-600^{\circ} \mathrm{C}$ for melteigite/alkali gabbro and $\sim 400^{\circ} \mathrm{C}$ for syenite. Critical assessment of other thermometric methods reveals a temperature of equilibration of $\sim 700^{\circ}-1350^{\circ} \mathrm{C}$ for ijolite-melteigite suite of rocks in contrast to a relatively lower equilibration temperature of $\sim 600^{\circ} \mathrm{C}$ for syenite. Geobarometric data based on pyroxene chemistry yield an equilibration pressure of $5.32-7.72 \mathrm{~kb}$ for ijolite, melteigite, alkali pyroxenite, alkali gabbro and nepheline syenite. The dominant syenite member of the intrusive plug records a much higher $(\sim 11 \mathrm{~kb})$ equilibration pressure indicating a deeper level of intrusion. Major oxide variations of constituent lithomembers with respect to differentiation index (D.I.) corroborate a normal magmatic differentiation. A prominent role of liquid immiscibility is envisaged from field geological, petrographic and petrochemical evidences. Tectonic discrimination diagrams involving clinopyroxene chemistry strongly suggest within plate alkaline affinity for the parental magma which is in conformity with the regional plume tectonics.

\section{Introduction}

The initial and waning stages of Continental Flood Basalt (CFB) volcanisms are marked by alkaline magmatic intrusions which represent various differentiating phases of the main flood basalt event (Basu et al 1993; Renne et al 1996; Ray and Pande 2001; Nambiar 2007). An association

Keywords. Alkaline complex; Sylhet Trap; thermometry; geobarometry; liquid immiscibility; plume tectonics. 
between alkaline magmatism and carbonatite is well documented (Le Bas 1987, 1989; Gittins 1989; Bailey 1995; Bell et al 1998) and it is spatially and temporally associated with continental flood basalts (CFB), which are generally interpreted as manifestation of plume activities (Heaman et al 2002; Bell 2001, 2002).

In northeastern India, the Sylhet Traps are exposed in a narrow, $80 \mathrm{~km}$ long and $4 \mathrm{~km}$ wide east-west trending belt. This belt is part of BengalSylhet-Rajmahal flood basalt province and is located along the southern margin of the Shillong Plateau having a maximum thickness of 550-650 m (Mazumder 1986). Baksi (1995) has considered Sylhet Traps to mark the beginning of Kerguelen plume activities beneath the Indian Plate (Nambiar 1988; Baksi 1995; Nandy 2001; Mahoney et al 1983; Veena et al 1998; Kent et al 2002) around $117 \mathrm{Ma}$. The Kerguelen hotspot was situated close to the eastern Indian margin 100-120 Ma ago and was responsible for the extrusion of Rajmahal basalts exposed over the Gondwana Supergroup and Sylhet basalts and related alkaline-carbonatite magmatism of Shillong Plateau (Coffin et al 2002; Kent et al 2002).

The Shillong Plateau of northeastern India underwent an extensive basic and alkalinecarbonatite magmatic activity during early Cretaceous (Kumar et al 1996; Srivastava et al 2005). A spatial and temporal association of this magmatism with Kerguelen hotspot-mantle plume system of Indian Ocean has been proposed (Ray et al 1999; Kent et al 2002). The ultramafic-mafic-alkalinecarbonatite intrusive complexes representing late stage differentiation of Sylhet Trap magmas occur as small, isolated, circular bodies along a NE trending zone in Shillong Plateau (Mamallan et al 1994; Srivastava and Sinha 2004, 2007) (figure 1). One important differentiation centre is represented by the Samchampi $\left(26^{\circ} 13^{\prime} \mathrm{N}: 93^{\circ} 18^{\prime} \mathrm{E}\right)$-Samteran $\left(26^{\circ} 11^{\prime} \mathrm{N}: 93^{\circ} 25^{\prime} \mathrm{E}\right)$ alkaline complex, which occurs as a near circular, plug-like [funnel shaped intrusive magmatic bodies, commonly emplaced into the brittle upper crust, elliptical and subcircular in cross section, are called plugs (Best 2003)] in intrusive body emplaced into the Precambrian basement gneisses of the Mikir Hills, in the Karbi-Anglong district of Assam (Kumar et al 1989; Nag et al 1999). The intrusions, emplaced in a granitic host rock, span a broad compositional lithospectrum embracing syenites (covering large part of the area), ultramafics (alkali pyroxenite), mafics (ijolite, melteigite, shonkinite, malignite), carbonatite, vanadium bearing titaniferous magnetite ore bodies. Small pockets of phosphatic, non-clastic sedimentary rocks overlie the pluton. A zone of fenitization encircles the complex.
Till date for the Samchampi-Samteran alkaline complex, only rudimentary petrological data are available in the literature (Kumar et al 1989; Nag et al 1999). Knowledge of mineral chemistry for different lithotypes and the genetic correlation among them is unknown. Here we report for the first time, mineral chemical compositions from the rocks of the Samchampi-Samteran alkaline complex to decipher the $\mathrm{P}-\mathrm{T}$ condition of equilibration of different constituent lithomembers of the area. Mineral chemical parameters have been considered to delineate the tectonic setting of the complex. Whole rock geochemical data (after Kumar et al 1989; Nag et al 1999) have been used to describe the geochemical characters and petrogenetic aspects of the intrusive complex. In this paper we combine field evidences, mineralogical and geochemical observations to present a cogent petrogenetic model which in turn would be useful for understanding broad tectonic setting of this type of differentiation centres present in NE India possibly linked up with Sylhet Traps.

\section{Geological overview: Mode of occurrence and field relations}

Alkaline magmatism is mostly concentrated in relatively stable, intracratonic platforms facilitated by major lithospheric uparch or lineaments. The emplacement and distribution of ultramafic-maficalkaline-carbonatite complexes are controlled by deep-seated faults and rift-like extension zones with abruptly decreasing thickness of the continental lithosphere (Frolov 1973; Woolley 1989). The Shillong Plateau forms a part of the NE extremity of the Indian peninsular shield. It is roughly rectangular in shape, covers an area of about $40,000 \mathrm{~km}^{2}$ and is considered as an uplifted horst-like feature, bounded by the E-W trending Dauki and Brahmaputra fault systems in the south and the north, respectively. The western and eastern fringes of the plateau are bordered by the $\mathrm{N}-\mathrm{S}$ trending Jamuna fault system and the NW-SE trending Kopili fracture zone respectively, the latter separating the Shillong Plateau from the Mikir Hills (Evans 1964; Desikachar 1974; Nandy 1980, 2001; Acharya et al 1986; Gupta and Sen 1988). Linear fracture patterns along the Assam-Meghalaya belt, directed towards NESW and ENE-WSW and subparallel to Kalyani Lineament and Sarhed fault (figure 2) (Nandy 1980, 2001; Nag et al 1999) are loci of major alkali emplacements and seismic activity. The Samchampi $\left(26^{\circ} 13^{\prime} \mathrm{N}\right.$ : $\left.93^{\circ} 18^{\prime} \mathrm{E}\right)$-Samteran $\left(26^{\circ} 11^{\prime} \mathrm{N}\right.$ : $93^{\circ} 25^{\prime} \mathrm{E}$ ) alkaline complex occurs as a near circular plug-like intrusion within the Precambrian granite gneisses of Mikir Hills in Karbi-Anglong district 


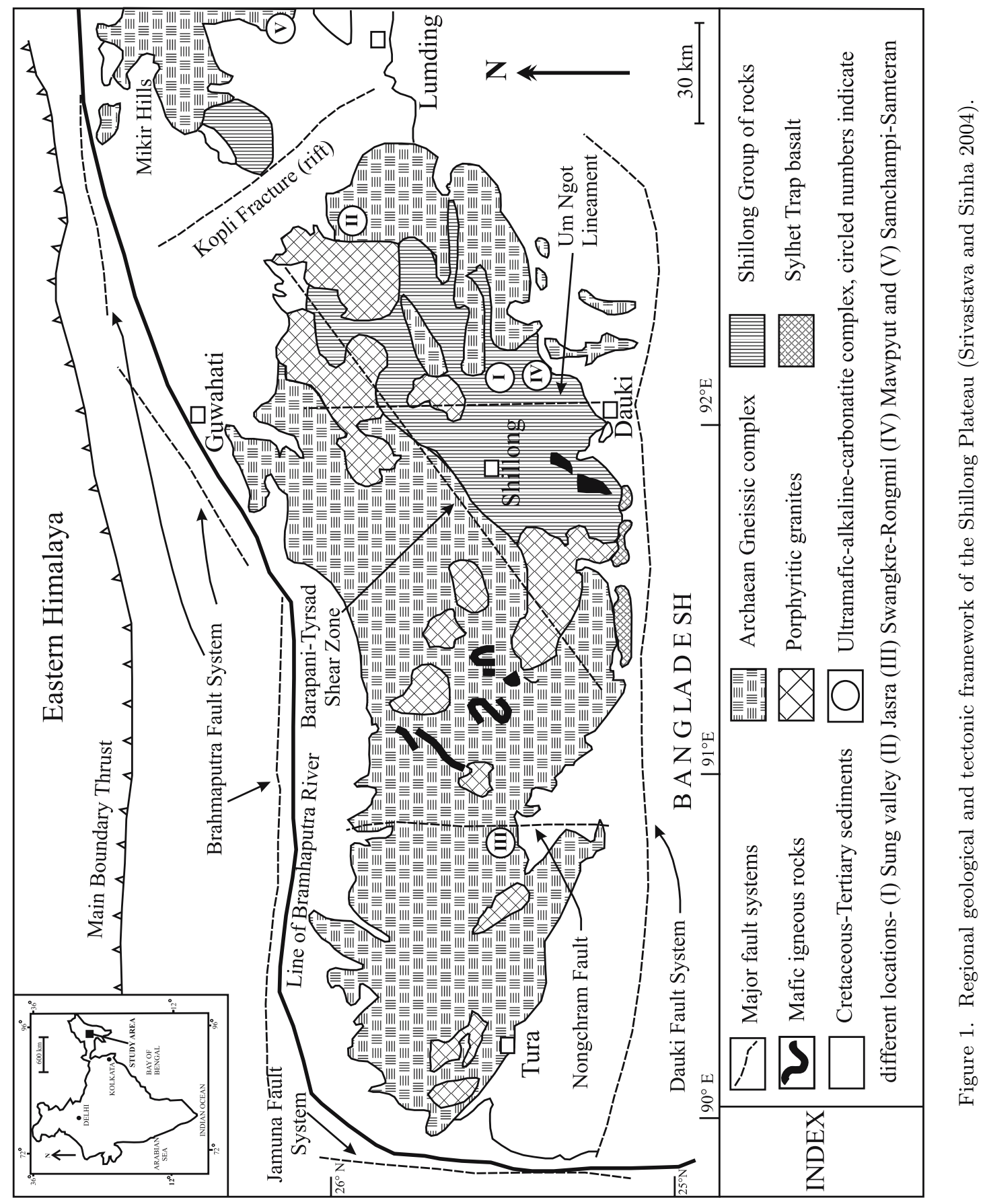




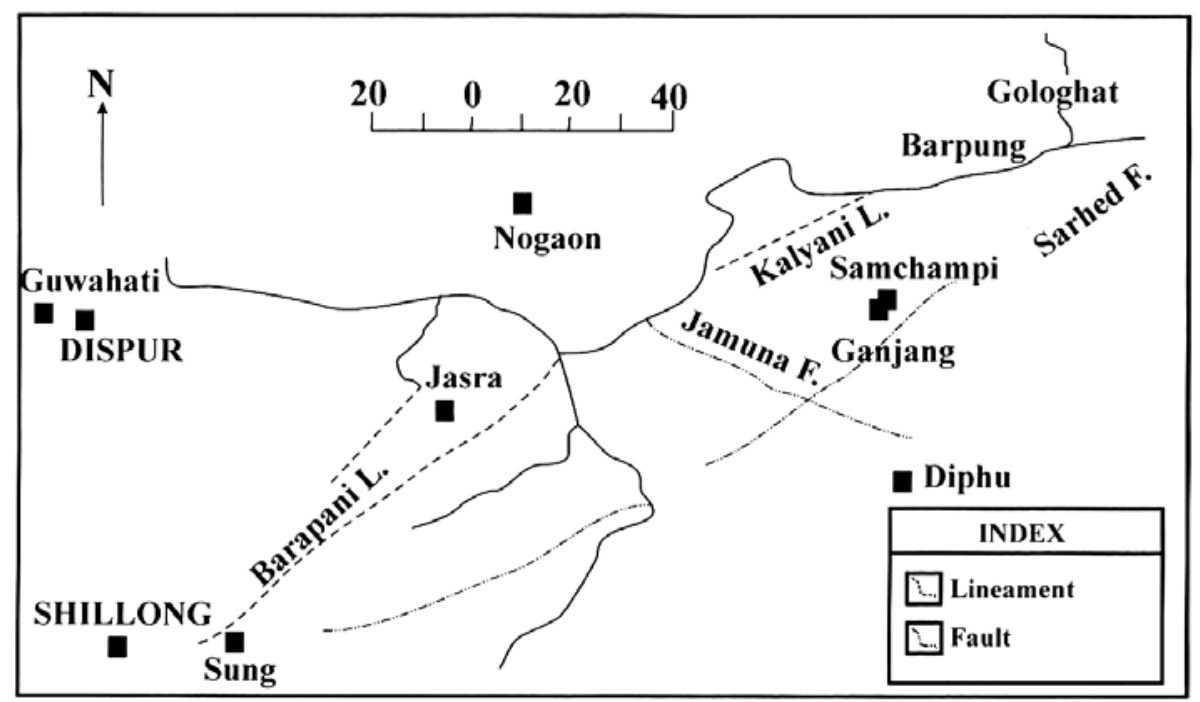

Figure 2. Map featuring major lineaments and faults defining the alkaline complexes of Assam-Meghalaya belt (after Nag et al 1999).

of Assam, northeastern India and it is linked with some of the major structural features of northeastern India. The pluton was emplaced at about $15 \mathrm{~km}$ south of the E-W trending Kalyani Lineament (figure 2). This lineament possibly merges with the deep-seated regional fracture defined by Barapani Lineament (figure 2) (Das et al 1995; Nag et al 1999) further to the west. The geological framework of the Samchampi-Samteran alkaline complex is given in figure 3 . The intrusive complex comprises a broad compositional lithospectrum including syenite, ijolite-melteigite, alkali pyroxenite, alkali gabbro, nepheline syenite and carbonatite. Syenite constitutes the dominant lithomember of the complex within which the ijolite-melteigite suite of rocks has been emplaced with an arcuate outcrop pattern (figure 3). Field observations record a sharp contact between syenite and ijolite-melteigite rocks. Alkali pyroxenite and alkali gabbro occur as inliers within the ijolite-melteigite suite and the field relation suggests that they are older than the latter. In the field, lumpy vanadium bearing titaniferous magnetite ore bodies are exposed as isolated intrusives within the syenite host rocks (figure 3). Dykes and dykelets of nepheline syenite and carbonatite occur as later intrusives cutting across the syenite and ijolite-melteigite rocks. Their spatial relationship suggests that they represent the youngest intrusive lithomember of the complex.

\section{Petrography and mineralogy}

Distinct petrographic features of the constituent lithomembers of the complex are described below.

\subsection{Syenite}

Megascopically, syenites are hard, massive and leucocratic. K-feldspar, plagioclase, clinopyroxene, amphibole and carbonates constitute the essential mineral composition of the rocks, while biotite, sphene, apatite and opaque occur as accessory minerals. In syenite, clinopyroxene is in the range $\mathrm{Wo}_{38-48} \mathrm{En}_{17-35} \mathrm{Fs}_{17-45}$ and composition of $\mathrm{K}$-feldspar shows the range $\mathrm{Or}_{70-96} \mathrm{Ab}_{5-28} \mathrm{An}_{0-2}$. In some samples, garnet is present as a major constituent. In sample SAJ 4A (syenite) clinopyroxene grains occur as inclusion within garnet ( $\mathrm{And}_{91-96} \mathrm{Gr}_{1-6} \mathrm{Py}_{0.7-1.2} \mathrm{Sp}_{1.4-1.6} \mathrm{Uv}_{0.16-0.4}$ ) (figure 4a) suggesting the earlier origin of clinopyroxene and replacement of clinopyroxene by garnet possibly due to metasomatic reactions. Biotite grains are formed at the expense of clinopyroxene grains. The rock is holocrystalline, coarse grained, equigranular and shows an overall hypidiomorphic granular texture with local development of perthitic intergrowths. Composition of plagioclase blebs in perthite is $\mathrm{Or}_{1} \mathrm{Ab}_{81} \mathrm{An}_{18}$. Modal compositions of the representative samples of this rock type (table 1) occupy the field of 'Alkali feldspar syenite' in the $\mathrm{Q}-\mathrm{A}-\mathrm{P}$ diagram (Streckeisen 1976).

\subsection{Ijolite-melteigite suite of rocks}

Ijolite-melteigite rocks of the investigated area are hard, massive and dominantly melanocratic in hand specimen. Nepheline and clinopyroxene represent essential mineralogy of these rocks. In some samples, biotite, garnet and carbonates are present as major constituents. Subordinate 


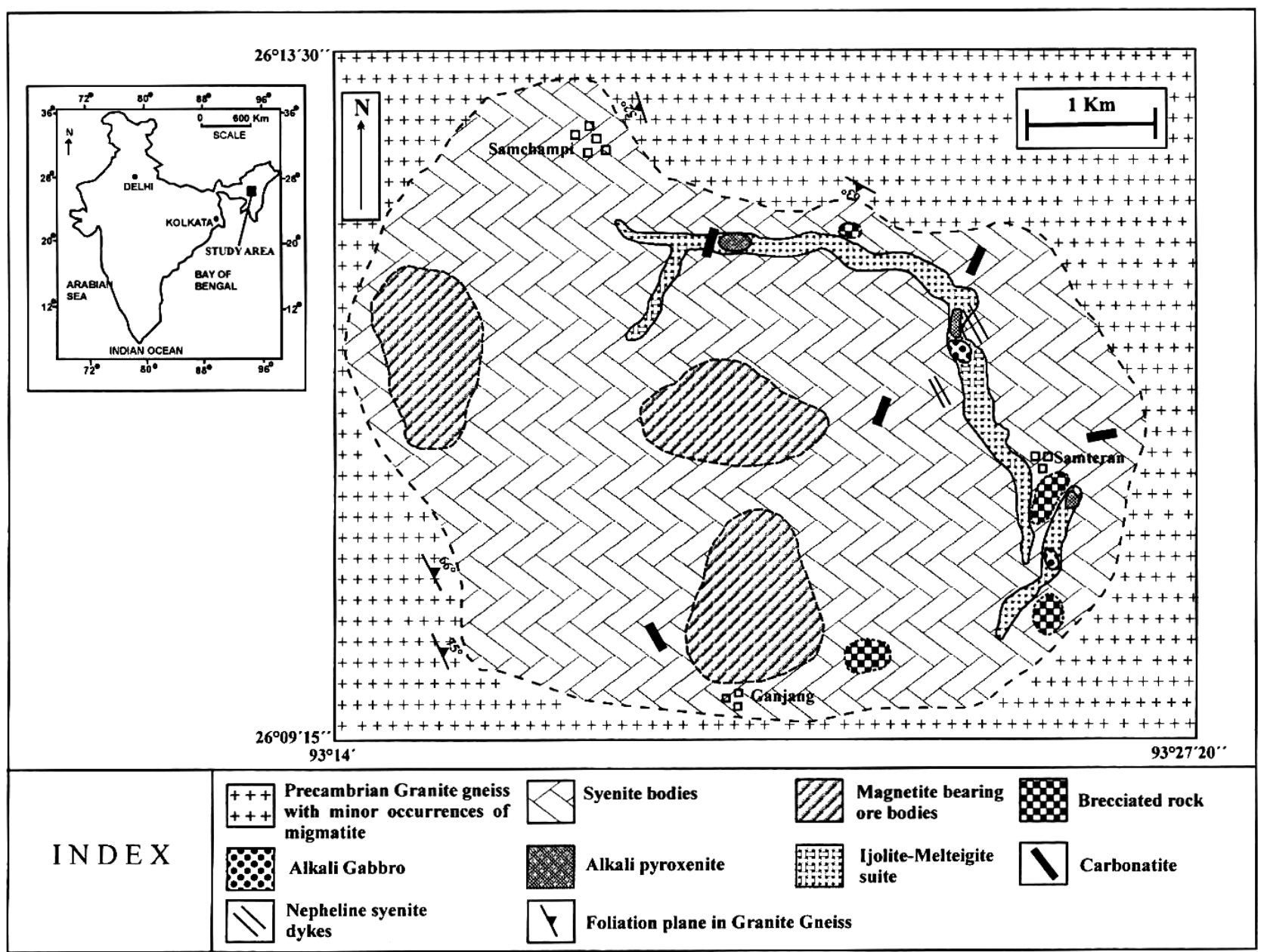

Figure 3. Geological map of Samchampi-Samteran ultramafic-mafic-alkaline-carbonatite complex. Inset map shows location of study area in the map of India.

amounts of K-feldspar, sphene, apatite and opaque represent the accessory mineral phases. Biotite grains have formed along the boundaries of clinopyroxene in some samples. In ijolite-melteigite rocks, clinopyroxene composition shows the range $\mathrm{Wo}_{45-48} \mathrm{En}_{30-36} \mathrm{Fs}_{15-26}$ with $\mathrm{mg} \#$ ranging from 0.68 to 0.83 . K-feldspar in ijolite (SAJ 3) shows the composition $\mathrm{Or}_{98} \mathrm{Ab}_{1.7} \mathrm{An}_{0.3}$. In sample SAJ 3 (ijolite), nepheline occurs as distinct inclusion within clinoproxene (figure 4b) while in sample SAJ 17 (melteigite), clinopyroxene occurs as inclusion within nepheline (figure 4c). These distinct petrographic features suggest that in ijolitemelteigite rocks, two generations of clinopyroxene are present. The earlier formed clinopyroxene occurs as inclusion within nepheline while the late formed clinopyroxene contains inclusion of nepheline in it. Garnet grains, in some cases, have developed at the expense of both clinopyroxene and biotite grains. In melteigite (SAJ 17) compostion of garnet is $\mathrm{And}_{83} \mathrm{Gr}_{9} \mathrm{Py}_{6} \mathrm{Sp}_{1.8} \mathrm{Uv}_{0.2}$. The ijolite-melteigite rocks are holocrystalline, coarse grained and show an overall hypidiomorphic granular texture. Local development of poikilitic texture has also been observed where clinopyroxene grains are enclosed by relatively larger plate of nepheline. In some places, intergrowth texture is exhibited by vermicular nepheline grains intergrown within K-feldspar base (figure 4d). The independent nepheline grains locally maintain optical continuity with the vermicular nepheline intergrowths suggesting that K-feldspar has replaced nepheline in these rocks. Similar K-feldsparnepheline replacement phenomenon has been described from Khariar alkaline complex of Orissa, India (Madhavan et al 1989). Representative modal compositions (table 1) occupy the FeldspathoidAlkali feldspar $(\mathrm{F}-\mathrm{A})$ arm of the $\mathrm{F}-\mathrm{A}-\mathrm{P}$ triangular diagram and the plots correspond to the field of 'Foidolites' (Streckeisen 1976). Further consideration of colour index (C.I.), modal mafic and foid (vol.\%) indicates that both ijolite and melteigite varieties are present, following IUGS classification scheme for foidal mafic rocks (Streckeisen 1976). 

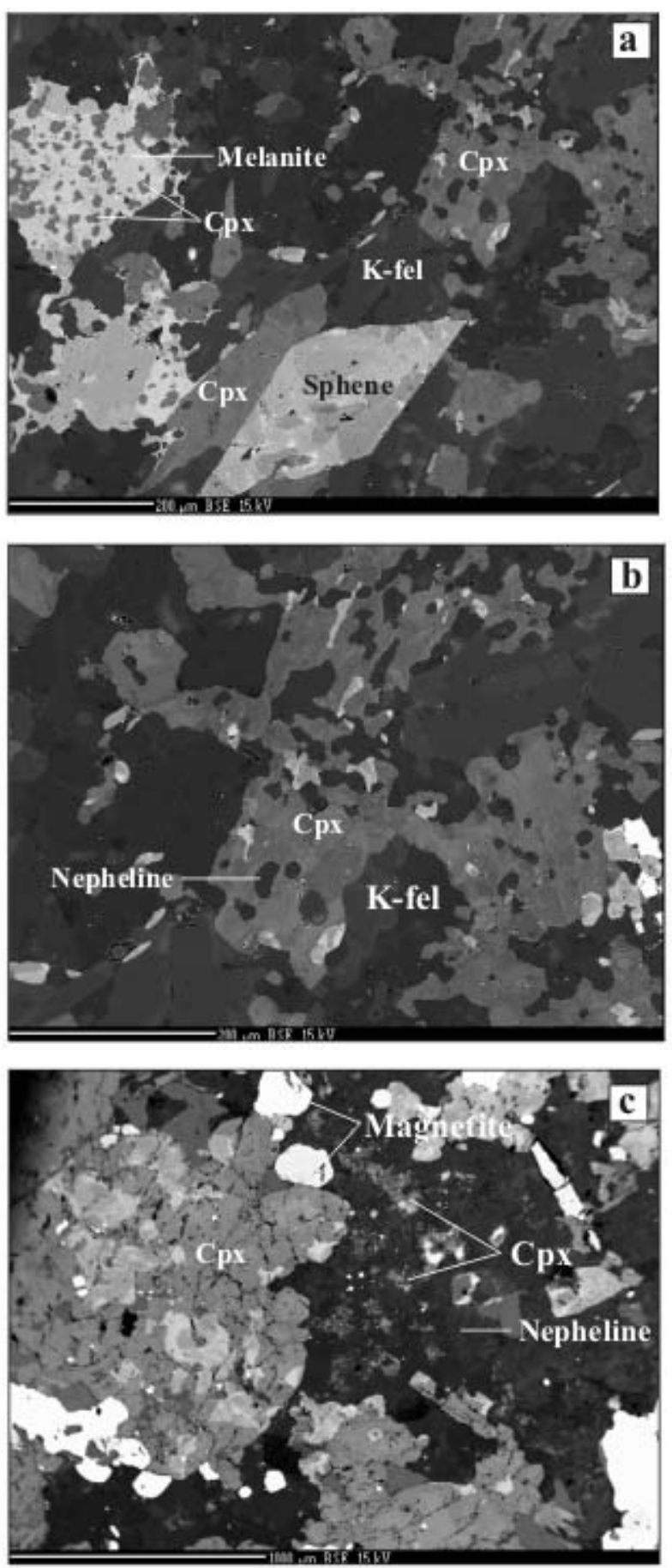

Figure 4. (a) BSE image showing inclusion clinopyroxene $(\mathrm{Cpx})$ within melanite in syenite. Occurrence of K-feldspar and sphene is also noted. (b) BSE image showing inclusion of nepheline within clinopyroxene $(\mathrm{Cpx})$ in ijolite. (c) BSE image showing inclusion clinopyroxene (Cpx) within nepheline in melteigite. Occurrence of magnetite along the grain boundary of Cpx is also noted.

\subsection{Alkali pyroxenite}

Megascopically, alkali pyroxenite rocks are hard, massive and melanocratic. They are holocrystalline, coarse grained and equigranular. Clinopyroxene occurs as essential and the most dominant mineral constituent with the compositional range $\mathrm{Wo}_{41-45} \mathrm{En}_{33-35} \mathrm{Fs}_{20-26}$. Accessory minerals include $\mathrm{K}$-feldspar $\left(\mathrm{Or}_{88.8} \mathrm{Ab}_{11.2}\right)$, nepheline and carbonates with sphene, apatite, biotite and opaque. These rocks show an overall hypidiomorphic granular texture but cumulus texture is locally developed. Clinopyroxene is the cumulus phase and nepheline occurs in the intervening spaces representing the intercumulus phase. Average modal compositions of the representative alkali pyroxenite samples have been presented in table 1 .

\subsection{Alkali gabbro}

The alkali gabbro rock bodies are hard, massive and melanocratic in hand specimen. These rocks are essentially composed of clinopyroxene $\left(\mathrm{Wo}_{46-47}\right.$ $\left.\mathrm{En}_{37-40} \mathrm{Fs}_{14-15}\right), \quad \mathrm{K}$-feldspar $\left(\mathrm{Or}_{97.5} \mathrm{Ab}_{2.5}\right)$ and nepheline with lesser amounts of biotite. Carbonates are present in some samples. Accessory phases include subordinate amounts of sphene, apatite and opaque. The overall textural pattern is defined by a poikilitic relationship where clinopyroxene is enclosed within K-feldspar (figure 4e). The modal compositions (table 1), occupy the $\mathrm{F}-\mathrm{A}$ arm of the $\mathrm{F}-\mathrm{A}-\mathrm{P}$ triangle and correspond to the field of 'Foid syenite' (Streckeisen 1976). Following Streckeisen's (1976) criteria, the alkali gabbro may further be subdivided into malignite and shonkinite (table 1).

\subsection{Minor intrusives: Nepheline syenite and carbonatite}

Megascopically, nepheline syenites are hard, massive and mesocratic. The essential mineralogical composition is represented by $\mathrm{K}$-feldspar $\left(\mathrm{Or}_{83} \mathrm{Ab}_{17}\right)$ and nepheline. Clinopyroxene $\left(\mathrm{Wo}_{43-44}\right.$ $\left.\mathrm{En}_{31-32} \mathrm{Fs}_{25-26}\right)$ occurs as major mafic constituent. Subordinate amounts of biotite, carbonates, sphene and apatite represent the accessory phases. In some samples, minor occurrence of ferrugenous alteration of mafic minerals has been noticed. An overall hypidiomorphic granular textural pattern has been documented. Poikilitic texture is developed locally, where clinopyroxene grains are enclosed by nepheline depicting a poikilitic relationship. The modal plots occupy the $\mathrm{F}-\mathrm{A}$ arm of the $\mathrm{F}-\mathrm{A}-\mathrm{P}$ triangle and belong to the field of 'Foid syenite' where foid is being represented by nepheline (Streckeisen 1976).

Carbonatites are essentially composed of carbonate minerals with apatite and minor amounts of biotite and clinopyroxene. Opaque minerals represent the accessory phase. These rocks are coarse grained and sovitic. Carbonate grains are arranged in a granular mosaic pattern defining an overall hypidiomorphic granular texture (figure 4f). These 
Table 1. Average modal (vol.\%) composition of the different intrusives.

\begin{tabular}{|c|c|c|c|c|c|c|c|c|c|}
\hline & \multirow[b]{2}{*}{ Syenite } & \multirow{2}{*}{$\begin{array}{c}\text { Melanite } \\
\text { bearing } \\
\text { syenite }\end{array}$} & \multirow[b]{2}{*}{ Ijolite } & \multirow[b]{2}{*}{ Melteigite } & \multirow{2}{*}{$\begin{array}{c}\text { Alkali } \\
\text { pyroxenite }\end{array}$} & \multicolumn{2}{|c|}{ Alkali gabbro } & \multirow{2}{*}{$\begin{array}{l}\text { Nepheline } \\
\text { syenite }\end{array}$} & \multirow[b]{2}{*}{ Carbonatite } \\
\hline & & & & & & Shonkinite & Malignite & & \\
\hline & ${ }^{*} n=9$ & ${ }^{*} n=3$ & ${ }^{*} n=8$ & ${ }^{*} n=5$ & ${ }^{*} n=3$ & ${ }^{*} n=2$ & ${ }^{*} n=2$ & ${ }^{*} n=4$ & ${ }^{*} n=4$ \\
\hline K-feldspar & 69.6 & 73.3 & 8.2 & - & 1.2 & 20.4 & 27.9 & 56.6 & - \\
\hline Plagioclase & 3.1 & 5.8 & - & - & - & - & - & - & - \\
\hline Nepheline & - & - & 44.7 & 17.9 & 2.8 & 10.2 & 20.3 & 22.7 & - \\
\hline Clinopyroxene & 10.3 & Trace & 33.3 & 56.7 & 92.6 & 64.2 & 33.7 & 17.9 & 6.5 \\
\hline Amphibole & 8.2 & 7.4 & 0.3 & - & - & - & - & - & - \\
\hline Biotite & 1.8 & - & 9.4 & 5.9 & 0.5 & 2.9 & 11.5 & 0.7 & 11.4 \\
\hline Garnet & - & 10.7 & - & 6.3 & - & - & - & - & - \\
\hline Carbonate & 3.6 & 1.2 & 1.4 & 10.4 & 1.7 & - & 1.4 & 1.7 & 71.3 \\
\hline Sphene & 1.3 & 0.9 & 1.0 & 0.4 & 0.8 & 2.3 & 1.7 & - & - \\
\hline Apatite & 0.2 & 0.4 & 0.8 & 0.7 & 0.2 & 0.3 & 0.8 & 0.3 & 8.5 \\
\hline Opaque & 1.9 & 0.3 & 1.0 & 1.7 & 0.1 & - & 2.7 & 0.1 & 2.4 \\
\hline
\end{tabular}

${ }^{*} n=$ number of data.

rocks have $>50 \%$ vol. of carbonate minerals (conforming to general classification scheme proposed by Middlemost 1985, p. 222).

\subsection{Vanadium bearing tiataniferous magnetite ore bodies}

Megascopically, the rocks are hard, massive and deep brown in colour. These rocks are dominantly composed of magnetite associated with hematite. Pronounced effects of martitization are evident where magnetite has been oxidized and replaced by haematite which occurs as lamellae. Box-work type intergrowth textural pattern has been observed in martitized grains.

\section{Mineral chemistry}

Quantitative chemical analyses of constituent mineral phases have been undertaken at Department of Earth, Atmospheric and Planetary Sciences, Massachusetts Institute of Technology, USA. Major element compositions of constituent minerals were analysed by wavelength dispersive spectrometry (WDS) on a JEOL JXA-733 electron probe micro analyzer (WDS) with computer control. A fully focused beam operating with beam energy of $15 \mathrm{keV}$ and a probe current of $300 \mathrm{nA}$ was used. Typical counting times were 20-40 seconds and $1 \sigma$ standard deviations of the counts were $0.5-1 \%$. Synthetic as well as natural standards were used during the analyses.

\subsection{Pyroxene}

The mineral chemical data obtained from twelve pyroxene analyses representing the alkaline felsic (syenite, nepheline syenite) and alkaline maficultramafic (ijolite, melteigite, alkali gabbro and alkali pyroxenite) members of the presently investigated Samchampi-Samteram Complex have been furnished in table 2 . The pyroxenes are classified on the basis of ' $Q$ ' and ' $\mathrm{J}$ ' relations (Morimoto et al 1988). In the Q-J classificatory diagram (figure 5a; Morimoto 1989), the pyroxenes of syenite, melteigite and alkali gabbro occupy the 'Quad' field and are thus Quad, whereas the pyroxenes from alkali pyroxenite, ijolite and nepheline syenite fall in the field of $\mathrm{Ca}-\mathrm{Na}$ pyroxenes and are designated as non-Quad pyroxenes. The Quad pyroxenes are mostly diopside in composition (figure 5b) while the non-Quad pyroxenes largely cluster in the aegirine-augite field (figure 5c). Further in the Di-Hd-En-Fs diagram (Morimoto et al 1988; Rock 1990), pyroxene compositions corresponding to melteigite, alkali gabbro and syenite follow the 'alkaline trend' (Dobosi 1987) while lone plot of pyroxene corresponding to syenite follows the 'mildly alkaline trend' (Dorais et al 1991) (figure 5d).

\subsection{Feldspar}

Altogether eleven mineral-chemical analyses of feldspar from three constituent lithomembers of the study area, viz., syenite, alkali pyroxenite and alkali gabbro have been presented in table 3. Out of eleven analyses, nine correspond to K-feldspar while rest two represent plagioclase. Alkali feldspar data are available for syenite, ijolite, alkali pyroxenite, alkali gabbro and neplheline syenite. In the $\mathrm{Or}-\mathrm{Ab}-\mathrm{An}$ triangular diagram (figure 6), the data-plots corresponding to syenite, ijolite, alkali pyroxenite, alkali gabbro and nepheline syenite reflect an orthoclase composition. 
Table 2. EPM analyses of pyroxene.

\begin{tabular}{|c|c|c|c|c|c|}
\hline \multirow{2}{*}{$\frac{\text { Sample }}{\text { Analysis }}$} & \multicolumn{2}{|c|}{ SAJ 11} & \multirow{2}{*}{$\frac{\text { SAJ } 17}{3}$} & \multicolumn{2}{|c|}{ SAJ 19} \\
\hline & 1 & 2 & & 4 & 5 \\
\hline Rock type & $\mathrm{AP}$ & $\mathrm{AP}$ & $\mathrm{M}$ & M & M \\
\hline $\mathrm{SiO}_{2}$ & 52.39 & 53.21 & 49.87 & 51.28 & 53.24 \\
\hline $\mathrm{TiO}_{2}$ & 0.21 & 0.16 & 0.68 & 0.38 & 0.09 \\
\hline $\mathrm{Al}_{2} \mathrm{O}_{3}$ & 0.91 & 0.69 & 2.55 & 2.59 & 0.51 \\
\hline $\mathrm{FeO}$ & 4.85 & 3.33 & 7.72 & 4.97 & 6.90 \\
\hline $\mathrm{Fe}_{2} \mathrm{O}_{3}$ & 6.74 & 10.47 & 6.06 & 4.40 & 2.84 \\
\hline $\mathrm{Cr}_{2} \mathrm{O}_{3}$ & 0.07 & 0.08 & 0.02 & 0.01 & 0.03 \\
\hline $\mathrm{MnO}$ & 0.54 & 0.53 & 0.51 & 0.28 & 0.55 \\
\hline $\mathrm{MgO}$ & 11.10 & 9.62 & 9.26 & 12.15 & 12.23 \\
\hline $\mathrm{CaO}$ & 19.78 & 16.29 & 20.08 & 22.39 & 22.24 \\
\hline $\mathrm{Na}_{2} \mathrm{O}$ & 2.66 & 4.72 & 2.11 & 1.31 & 1.29 \\
\hline Total & 99.25 & 99.10 & 98.86 & 99.75 & 99.91 \\
\hline \multicolumn{6}{|l|}{$0=6$} \\
\hline TSi & 1.974 & 2.003 & 1.913 & 1.918 & 1.993 \\
\hline TAl & 0.026 & - & 0.087 & 0.082 & 0.007 \\
\hline $\mathrm{TFe}_{3}$ & - & - & - & - & - \\
\hline $\mathrm{M}_{1} \mathrm{Al}$ & 0.015 & 0.031 & 0.029 & 0.032 & 0.015 \\
\hline $\mathrm{M}_{1} \mathrm{Ti}$ & 0.006 & 0.004 & 0.020 & 0.011 & 0.002 \\
\hline $\mathrm{M}_{1} \mathrm{Fe}_{3}$ & 0.191 & 0.296 & 0.175 & 0.124 & 0.080 \\
\hline $\mathrm{M}_{1} \mathrm{Fe}_{2}$ & 0.153 & 0.105 & 0.247 & 0.155 & 0.216 \\
\hline $\mathrm{M}_{1} \mathrm{Cr}$ & 0.002 & 0.002 & 0.001 & - & 0.001 \\
\hline $\mathrm{M}_{1} \mathrm{Mg}$ & 0.624 & 0.540 & 0.530 & 0.677 & 0.682 \\
\hline $\mathrm{M}_{2} \mathrm{Fe}_{2}$ & - & - & 0.001 & - & - \\
\hline $\mathrm{M}_{2} \mathrm{Mn}$ & 0.017 & 0.017 & 0.017 & 0.009 & 0.017 \\
\hline $\mathrm{M}_{2} \mathrm{Ca}$ & 0.799 & 0.657 & 0.825 & 0.897 & 0.892 \\
\hline $\mathrm{M}_{2} \mathrm{Na}$ & 0.194 & 0.345 & 0.157 & 0.095 & 0.094 \\
\hline Sum_cat & 4 & 4 & 4 & 4 & 4 \\
\hline $\mathrm{Q}$ & 1.58 & 1.30 & 1.60 & 1.73 & 1.79 \\
\hline $\mathrm{J}$ & 0.39 & 0.69 & 0.31 & 0.19 & 0.19 \\
\hline WO & 44.79 & 40.68 & 46.01 & 48.17 & 47.25 \\
\hline $\mathrm{EN}$ & 34.97 & 33.43 & 29.52 & 36.37 & 36.15 \\
\hline FS & 20.24 & 25.89 & 24.47 & 15.46 & 16.60 \\
\hline WEF & 80.38 & 65.68 & 83.77 & 90.16 & 90.60 \\
\hline JD & 1.39 & 3.23 & 2.28 & 2.01 & 1.51 \\
\hline $\mathrm{AE}$ & 18.23 & 31.09 & 13.95 & 7.83 & 7.89 \\
\hline $\mathrm{mg} \#$ & 0.80 & 0.84 & 0.68 & 0.81 & 0.76 \\
\hline
\end{tabular}

AP: Alkali pyroxenite; M: Melteigite.

Plagioclase occurs in syenite as exsolution blebs within perthite. The analysed plagioclase of syenite corresponds to oligoclase species $\left(\mathrm{An}_{18.4}-\mathrm{An}_{18.5}\right)$ (table 3).

\subsection{Nepheline}

Nepheline data are available for melteigite and nepheline syenite (table 4). The analyzed nepheline compositions are characterized by a higher proportion of $\mathrm{Na}_{2} \mathrm{O}$ (ranging from 15.44-15.95 wt.\%) with relatively lesser amounts of $\mathrm{K}_{2} \mathrm{O}$ (ranging from 6.70-7.59 wt.\%), thereby reflecting an overall sodic nature. The $\mathrm{Na} / \mathrm{Na}+\mathrm{K}$ ratio of nepheline in both melteigite and nepheline syenite is identical (0.76).
Thus, the representative $\mathrm{Na} / \mathrm{Na}+\mathrm{K}$ ratios suggest a soda-rich, alkaline character of the SamchampiSamteran intrusives with prominent influence of silica-undersaturation (Dawson et al 1995).

\subsection{Biotite}

Representative electron probe compositions of biotite from four lithomembers of the study area, namely, ijolite, melteigite, alkali pyroxenite and nepheline syenite have been presented in table 5 . The mineral chemical data reveal overall uniform biotite chemistry in all the four lithotypes. The analyzed biotite compositions represent a characteristic higher proportion of $\mathrm{K}_{2} \mathrm{O}$ ranging from 
Table 2. (Continued).

\begin{tabular}{|c|c|c|c|c|c|c|c|}
\hline \multirow{2}{*}{$\frac{\text { Sample }}{\text { Analysis }}$} & \multicolumn{2}{|c|}{ SAJ 25} & \multirow{2}{*}{$\frac{\text { SAJ } 3}{8}$} & \multirow{2}{*}{$\frac{\text { SAJ } 47}{9}$} & \multirow{2}{*}{$\frac{\text { SAJ } 4 \mathrm{~A}}{10}$} & \multicolumn{2}{|c|}{ SAJ 4B } \\
\hline & 6 & 7 & & & & 11 & 12 \\
\hline Rock type & NS & NS & I & $\mathrm{S}$ & $\mathrm{S}$ & $\mathrm{AG}$ & $\mathrm{AG}$ \\
\hline $\mathrm{SiO}_{2}$ & 52.72 & 52.81 & 52.91 & 49.85 & 50.45 & 52.97 & 52.94 \\
\hline $\mathrm{TiO}_{2}$ & 0.51 & 0.60 & - & - & 0.52 & 0.25 & 0.29 \\
\hline $\mathrm{Al}_{2} \mathrm{O}_{3}$ & 0.47 & 0.47 & 0.46 & 0.64 & 1.06 & 0.67 & 0.63 \\
\hline $\mathrm{FeO}$ & 6.84 & 6.00 & 3.85 & 23.11 & 4.48 & 7.29 & 4.77 \\
\hline $\mathrm{Fe}_{2} \mathrm{O}_{3}$ & 7.24 & 8.60 & 8.21 & 1.65 & 5.99 & 1.44 & 3.37 \\
\hline $\mathrm{Cr}_{2} \mathrm{O}_{3}$ & 0.03 & 0.03 & - & - & 0.04 & 0.02 & - \\
\hline $\mathrm{MnO}$ & 0.38 & 0.43 & 0.58 & 1.79 & 0.43 & 0.56 & 0.47 \\
\hline $\mathrm{MgO}$ & 9.86 & 9.72 & 10.81 & 5.59 & 11.48 & 12.40 & 13.33 \\
\hline $\mathrm{CaO}$ & 18.87 & 18.42 & 19.80 & 17.58 & 22.09 & 21.98 & 21.43 \\
\hline $\mathrm{Na}_{2} \mathrm{O}$ & 3.13 & 3.52 & 3.06 & 0.41 & 1.54 & 1.17 & 1.53 \\
\hline Total & 100.04 & 100.61 & 99.68 & 100.61 & 98.07 & 98.75 & 98.77 \\
\hline \multicolumn{8}{|l|}{$O=6$} \\
\hline TSi & 1.987 & 1.979 & 1.985 & 1.981 & 1.931 & 2.000 & 1.986 \\
\hline TAl & 0.013 & 0.021 & 0.015 & 0.019 & 0.048 & - & 0.014 \\
\hline $\mathrm{TFe}_{3}$ & - & - & - & - & 0.021 & - & - \\
\hline $\mathrm{M}_{1} \mathrm{Al}$ & 0.007 & - & 0.006 & 0.011 & - & 0.030 & 0.014 \\
\hline $\mathrm{M}_{1} \mathrm{Ti}$ & 0.014 & 0.017 & - & - & 0.015 & 0.007 & 0.008 \\
\hline $\mathrm{M}_{1} \mathrm{Fe}_{3}$ & 0.205 & 0.242 & 0.232 & 0.049 & 0.152 & 0.041 & 0.095 \\
\hline $\mathrm{M}_{1} \mathrm{Fe}_{2}$ & 0.216 & 0.188 & 0.121 & 0.608 & 0.143 & 0.224 & 0.137 \\
\hline $\mathrm{M}_{1} \mathrm{Cr}$ & 0.001 & 0.001 & - & - & 0.001 & 0.001 & - \\
\hline $\mathrm{M}_{1} \mathrm{Mg}$ & 0.554 & 0.543 & 0.605 & 0.331 & 0.655 & 0.698 & 0.745 \\
\hline $\mathrm{M}_{2} \mathrm{Fe}_{2}$ & - & - & - & 0.160 & - & 0.007 & 0.012 \\
\hline $\mathrm{M}_{2} \mathrm{Mn}$ & 0.012 & 0.014 & 0.018 & 0.060 & 0.014 & 0.018 & 0.015 \\
\hline $\mathrm{M}_{2} \mathrm{Ca}$ & 0.762 & 0.740 & 0.796 & 0.749 & 0.906 & 0.889 & 0.861 \\
\hline $\mathrm{M}_{2} \mathrm{Na}$ & 0.229 & 0.256 & 0.223 & 0.031 & 0.114 & 0.086 & 0.111 \\
\hline Sum_cat & 4 & 4 & 4 & 4 & 4 & 4 & 4 \\
\hline $\mathrm{Q}$ & 1.53 & 1.47 & 1.52 & 1.85 & 1.71 & 1.82 & 1.76 \\
\hline $\mathrm{J}$ & 0.46 & 0.51 & 0.45 & 0.06 & 0.23 & 0.17 & 0.22 \\
\hline WO & 43.57 & 42.83 & 44.93 & 38.25 & 47.92 & 47.39 & 46.15 \\
\hline EN & 31.68 & 31.45 & 34.13 & 16.92 & 34.65 & 37.20 & 39.94 \\
\hline FS & 24.75 & 25.72 & 20.93 & 44.83 & 17.43 & 15.40 & 13.92 \\
\hline WEF & 77.14 & 74.37 & 77.57 & 96.83 & 88.29 & 91.44 & 88.83 \\
\hline JD & 0.80 & - & 0.55 & 0.59 & - & 3.60 & 1.43 \\
\hline $\mathrm{AE}$ & 22.06 & 25.63 & 21.88 & 2.58 & 11.71 & 4.96 & 9.73 \\
\hline $\mathrm{mg} \#$ & 0.72 & 0.74 & 0.83 & 0.35 & 0.82 & 0.76 & 0.84 \\
\hline
\end{tabular}

NS: Nepheline syenite; I: Ijolite; S: Syenite; AG: Alkali gabbro.

9.22-10.09 wt\%. The $(\mathrm{FeO} / \mathrm{FeO}+\mathrm{MgO})$ ratio in biotite ranges between 0.39 and 0.52 and according to Czamanske and Wones (1973), such a low value of $(\mathrm{FeO} / \mathrm{FeO}+\mathrm{MgO})$ reflects increasing $f \mathrm{O}_{2}$ conditions during magmatic evolution. The aluminium saturation index (A.S.I) \{i.e., $\left.\mathrm{Al}^{\text {total }} / \mathrm{Ca}+\mathrm{Na}+\mathrm{K}\right\}$ calculated from mineral chemical data shows an increasing trend (ranging from 1.0 to 1.4) (table 5) and indicates sufficient alumina activity in the crystallizing magma (Zen 1988). In the Eastonite-Siderophyllite-AnnitePhlogopite diagram (figure 7a; Deer et al 1979), the data points occupy the 'biotite' field. A plot of biotite compositions in $\mathrm{TiO}_{2}-\mathrm{FeO}+\mathrm{MnO}-\mathrm{MgO}$ triangular diagram (Nachit et al 2005) (figure 7c) suggests their secondary origin. This is consistent with petrographic observations as well.

\subsection{Garnet}

Representative compositions of garnet from syenite and melteigite of the Samchampi-Samteran complex have been furnished in table 6 . The analyzed garnet compositions depict a prominent enrichment in $\mathrm{CaO}$ (ranging from 31.52 to 32.08 wt\%) with lesser amounts of $\mathrm{Al}_{2} \mathrm{O}_{3}$ (ranging from 0.67 to $2.7 \mathrm{wt} \%$ ) and $\mathrm{Cr}_{2} \mathrm{O}_{3}$ (ranging from 0.04 to $0.09 \mathrm{wt} \%$ ). Most significantly, the Samchampi-Samteran garnets contain variable amounts of $\mathrm{TiO}_{2}$, ranging from 3.62 to $12.88 \mathrm{wt} \%$, 

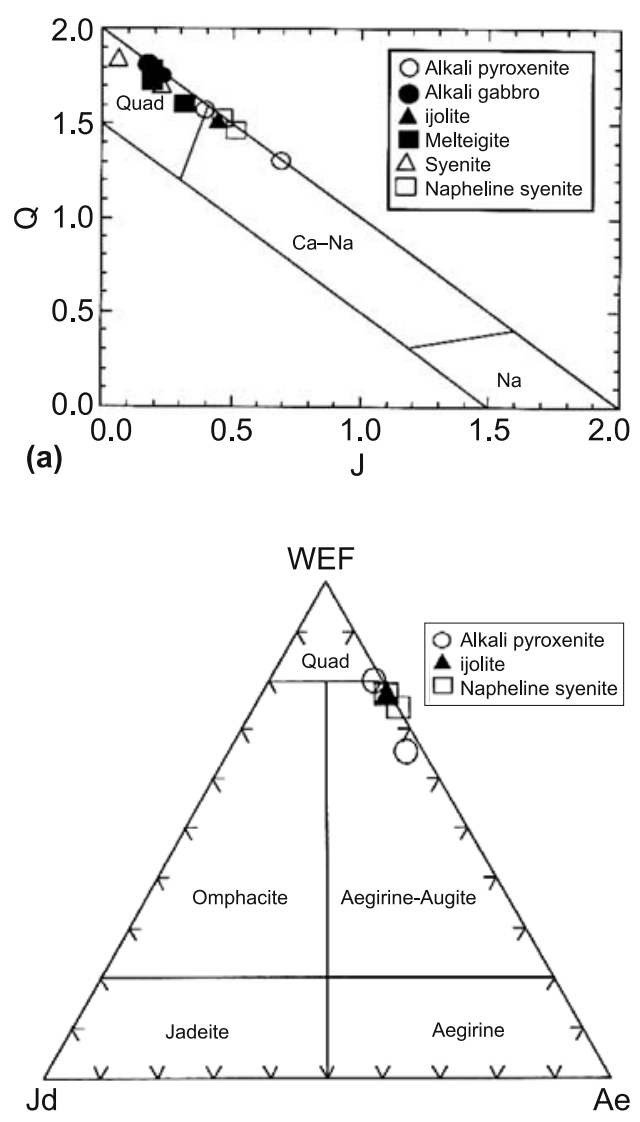

(c)

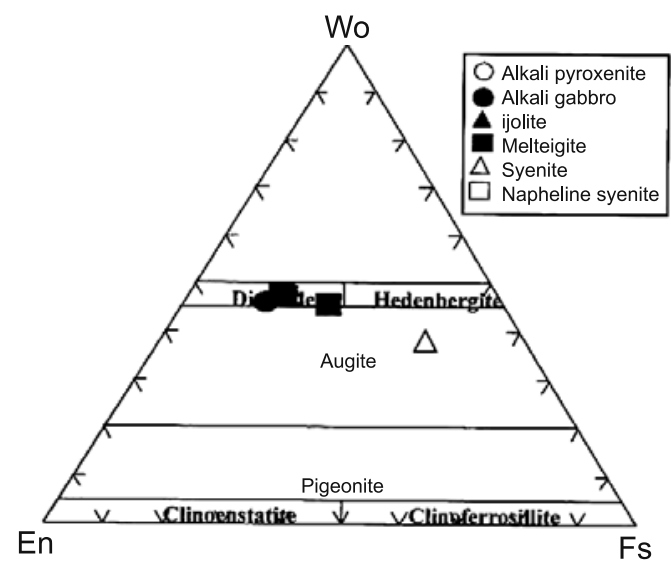

(b)

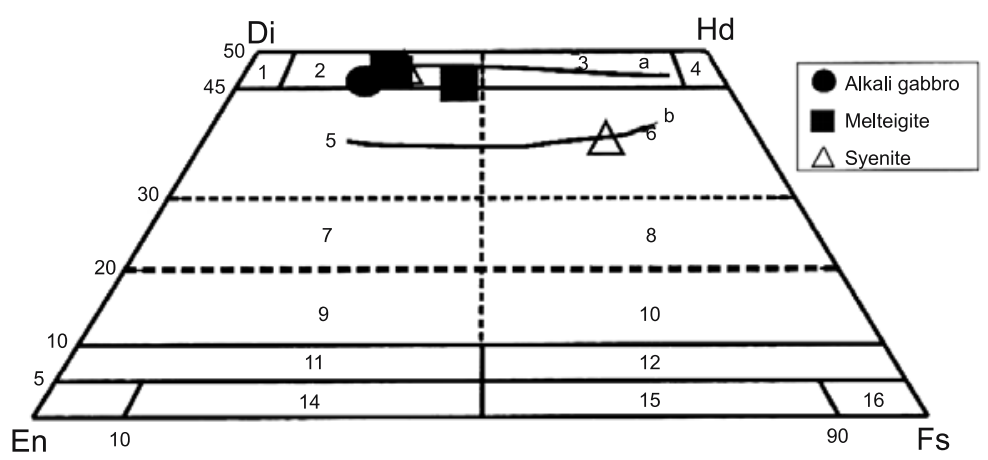

(d)

Figure 5. (a) Plot of investigated pyroxene compositions of Samchampi-Samteran complex in $\mathrm{Q}(\mathrm{Ca}+\mathrm{Mg}+\mathrm{Fe})-\mathrm{J}(2 \mathrm{Na})$ diagram (Morimoto et al 1989). (b) Plot of investigated pyroxene compositions of Samchampi-Samteran complex in Wo-En-Fs diagram (Morimoto et al 1989). (c) Plot of investigated pyroxene compositions of Samchampi-Samteran complex in WEF-En-Fs diagram (Morimoto et al 1989). (d) Clinopyroxene composition of Samchampi-Samteran complex in pyroxene quadrilateral (Morimoto et al 1988; Rock 1990). Variations shown as curved lines are (a) trend of pyroxenes from alkaline complexes (Dobosi 1987), (b) trend of pyroxene in mildly alkaline complexes (Dorais et al 1991). Fields are: (1) diopside, (2) ferroan diopside, (3) magnesian diopside, (4) hedenbergite, (5) magnesian-rich augite, (6) iron-rich augite, (7) subcalcic magnesian-rich augite, (8) subcalcic iron-rich augite, (9) calcian (magnesium-rich) pigeonite, (10) calcian (iron-rich) pigeonite, (11) magnesium-rich pigeonite, (12) iron-rich pigeonite, (13) clino-enstatite, (14) ferroan (clino) enstatite, (15) magnesian (clino) ferrosilite, and (16) clino-ferrosilite.

which reveals a unique compositional character. When the analyzed compositions of garnet are projected into the Spessartine+Pyrope-Ti-andraditeGrossular system, the compositional range corresponds to Ti-andradite (figure 8a) (Dingwell and Brearley 1985; Deer et al 1982). According to Deer et al (1992), Ti-rich andradites can be classified into melanite and schorlomite, depending on whether $\mathrm{Fe}^{3+}$ or Ti predominates in the octahedral site, placing the limit at approximately $15 \mathrm{wt} \%$ $\mathrm{TiO}_{2}$. Considering the classification parameters, the investigated Ti-andradite can be designated as melanite with a maximum $12.88 \mathrm{wt} \% \mathrm{TiO}_{2}$ (i.e., $\mathrm{TiO}_{2}$ content less than $\left.15 \%\right), \mathrm{Fe}^{3+}>\mathrm{Ti}, \mathrm{Al}<\mathrm{Fe}^{3+}$ (Deer et al 1992; Dawson et al 1995; Gwalani et al 2000) in all analyzed samples.

\subsection{Sphene}

Representative electron probe analyses of sphene from syenite, melteigite, alkali pyroxenite and alkali gabbro have been given in table 7 . The analyzed chemical compositions of sphene are broadly characterized by significant $\mathrm{TiO}_{2}$ (ranging from 33.45 to $37.27 \mathrm{wt} \%$ ) and $\mathrm{CaO}$ (ranging from 26.19 to $27.81 \mathrm{wt} \%$ ) contents. The relative proportions of $\mathrm{TiO}_{2}$ and $\mathrm{CaO}$ exhibit an overall uniform variation throughout the corresponding rock types.

\subsection{Opaque minerals}

Opaque minerals represent accessory phases in the constituent lithotypes of the present study 
Table 3. EPM analyses of feldspar.

\begin{tabular}{|c|c|c|c|c|c|c|c|c|c|c|c|}
\hline \multirow{2}{*}{$\frac{\text { Sample }}{\text { Analysis }}$} & \multirow{2}{*}{$\frac{\text { SAJ } 11}{1}$} & \multicolumn{2}{|c|}{ SAJ 25} & \multirow{2}{*}{$\frac{\text { SAJ } 3}{4}$} & \multirow{2}{*}{$\frac{\text { SAJ 4B }}{5}$} & \multirow{2}{*}{$\frac{\text { SAJ 4A }}{6}$} & \multicolumn{5}{|c|}{ SAJ 47} \\
\hline & & 2 & 3 & & & & 7 & 8 & 9 & $10^{*}$ & $11^{*}$ \\
\hline Rock type & $\mathrm{AP}$ & NS & NS & I & $\mathrm{AG}$ & $\mathrm{S}$ & $\mathrm{S}$ & S & S & $\mathrm{S}$ & $\mathrm{S}$ \\
\hline $\mathrm{SiO}_{2}$ & 65.32 & 65.34 & 66.14 & 65.76 & 65.40 & 65.46 & 65.96 & 66.61 & 66.13 & 64.90 & 64.94 \\
\hline $\mathrm{Al}_{2} \mathrm{O}_{3}$ & 18.88 & 18.55 & 18.08 & 18.87 & 18.54 & 18.54 & 19.23 & 19.30 & 19.77 & 23.28 & 23.16 \\
\hline $\mathrm{FeO}$ & 0.08 & 0.29 & 0.91 & 0.16 & 0.45 & 0.19 & 0.01 & 0.01 & 0.02 & 0.03 & 0.10 \\
\hline $\mathrm{MgO}$ & 0.01 & 0.01 & 0.01 & - & 0.02 & - & - & - & - & - & - \\
\hline $\mathrm{CaO}$ & - & - & - & 0.06 & - & - & 0.08 & 0.09 & 0.42 & 3.74 & 3.86 \\
\hline $\mathrm{Na}_{2} \mathrm{O}$ & 1.19 & 1.86 & 1.92 & 0.18 & 0.27 & 0.48 & 1.24 & 2.98 & 3.10 & 9.02 & 9.31 \\
\hline $\mathrm{K}_{2} \mathrm{O}$ & 14.35 & 13.65 & 13.76 & 15.73 & 15.63 & 15.66 & 14.16 & 13.67 & 12.04 & 0.16 & 0.24 \\
\hline Total & 99.83 & 99.69 & 100.82 & 100.76 & 100.11 & 100.34 & 100.67 & 102.67 & 101.48 & 101.13 & 101.61 \\
\hline \multicolumn{12}{|l|}{$\mathrm{O}=32$} \\
\hline $\mathrm{Si}$ & 11.984 & 12.000 & 12.049 & 12.005 & 12.037 & 12.016 & 11.976 & 11.903 & 11.864 & 11.296 & 11.280 \\
\hline $\mathrm{Al}$ & 4.079 & 4.012 & 3.879 & 4.057 & 3.975 & 4.008 & 4.112 & 4.062 & 4.177 & 4.772 & 4.737 \\
\hline $\mathrm{Fe}_{2}$ & 0.013 & 0.044 & 0.138 & 0.024 & 0.070 & 0.029 & 0.001 & 0.002 & 0.003 & 0.005 & 0.015 \\
\hline $\mathrm{Mg}$ & 0.002 & 0.003 & 0.003 & - & 0.005 & 0.001 & - & - & - & - & - \\
\hline $\mathrm{Ca}$ & - & - & - & 0.011 & - & - & 0.015 & 0.018 & 0.081 & 0.697 & 0.718 \\
\hline $\mathrm{Na}$ & 0.424 & 0.661 & 0.678 & 0.063 & 0.096 & 0.171 & 0.437 & 1.034 & 1.078 & 3.044 & 3.136 \\
\hline K & 3.359 & 3.198 & 3.198 & 3.663 & 3.670 & 3.667 & 3.280 & 3.116 & 2.756 & 0.036 & 0.053 \\
\hline Cations & 19.861 & 19.918 & 19.945 & 19.823 & 19.853 & 19.892 & 19.821 & 20.135 & 19.959 & 19.850 & 19.939 \\
\hline $\mathrm{X}$ & 16.063 & 16.012 & 15.928 & 16.062 & 16.012 & 16.024 & 16.088 & 15.965 & 16.041 & 16.068 & 16.017 \\
\hline Z & 3.798 & 3.906 & 4.017 & 3.761 & 3.841 & 3.868 & 3.733 & 4.170 & 3.918 & 3.782 & 3.922 \\
\hline $\mathrm{Ab}$ & 11.2 & 17.1 & 17.5 & 1.7 & 2.5 & 4.5 & 11.7 & 24.8 & 27.5 & 80.6 & 80.3 \\
\hline An & - & - & - & 0.3 & - & - & 0.4 & 0.4 & 2.1 & 18.5 & 18.4 \\
\hline Or & 88.8 & 82.9 & 82.5 & 98 & 97.5 & 95.5 & 87.9 & 74.8 & 70.4 & 1 & 1.4 \\
\hline
\end{tabular}

AP: Alkali pyroxenite; NS: Nepheline syenite; I: Ijolite; AG: Alkali gabbro; S: Syenite.

${ }^{*}$ Analysis no. 1-9 represent the probe data of K-feldspar of different lithotypes whereas analysis no. 10 and 11 represent the probe data of plagioclase from syenite.

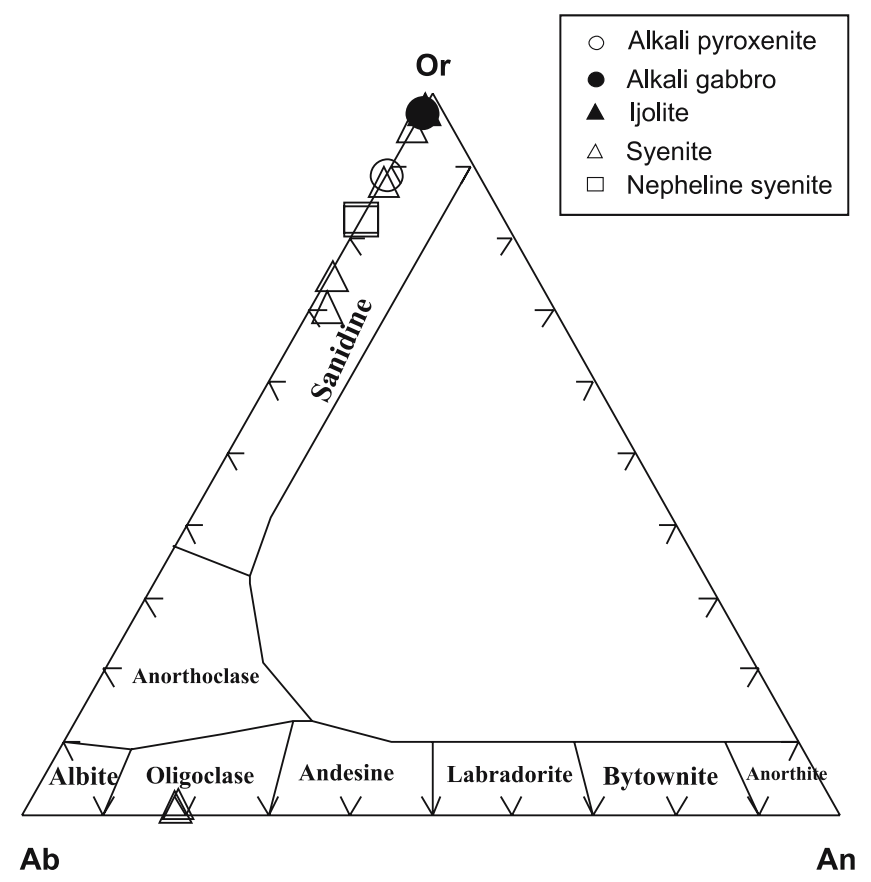

Figure 6. Plot of investigated feldspar compositions in Or-Ab-An triangular diagram.
Table 4. EPM analyses of nepheline.

\begin{tabular}{lccc}
\hline Sample & SAJ 19 & SAJ 19 & SAJ 25 \\
\hline Analyses & 1 & 2 & 3 \\
Rock type & $\mathrm{M}$ & $\mathrm{M}$ & $\mathrm{NS}$ \\
$\mathrm{SiO}_{2}$ & 41.85 & 41.85 & 42.92 \\
$\mathrm{Al}_{2} \mathrm{O}_{3}$ & 34.87 & 34.78 & 33.60 \\
$\mathrm{FeO}$ & 0.65 & 0.66 & 0.72 \\
$\mathrm{MgO}$ & 0.02 & - & - \\
$\mathrm{CaO}$ & 0.04 & 0.03 & - \\
$\mathrm{Na}_{2} \mathrm{O}$ & 15.44 & 15.68 & 15.95 \\
$\mathrm{~K}$ & $\mathrm{O}$ & 7.59 & 6.70 \\
$\mathrm{Total}$ & 7.58 & 100.59 & 99.91 \\
$\mathrm{Si}$ & 100.45 & 1.010 & 1.037 \\
$\mathrm{Al}$ & 1.010 & 0.989 & 0.957 \\
$\mathrm{Fe}$ & 0.992 & 0.013 & 0.015 \\
$\mathrm{Mg}$ & 0.013 & - & - \\
$\mathrm{Ca}$ & 0.001 & 0.001 & - \\
$\mathrm{Na}$ & 0.001 & 0.734 & 0.747 \\
$\mathrm{~K}$ & 0.722 & 0.234 & 0.207 \\
$\mathrm{CatTot}$ & 0.233 & 2.980 & 2.962 \\
$\mathrm{X}$ & 2.972 & 61.715 & 64.988 \\
$\mathrm{Y}$ & 61.571 & 45.183 & 74.272 \\
$\mathrm{Na} / \mathrm{Na}+\mathrm{K}$ & 45.621 & 0.76 & 0.78 \\
\hline $\mathrm{M}: \mathrm{Melteite}$ & 0.76 & &
\end{tabular}

M: Melteigite; NS: Nepheline syenite. 
Table 5. EPM analyses of biotite.

\begin{tabular}{|c|c|c|c|c|c|}
\hline \multirow{2}{*}{$\frac{\text { Sample }}{\text { Analysis }}$} & \multirow{2}{*}{$\frac{\text { SAJ } 11}{1}$} & \multicolumn{2}{|c|}{ SAJ 17} & \multirow{2}{*}{$\frac{\text { SAJ } 25}{4}$} & \multirow{2}{*}{$\frac{\text { SAJ } 3}{5}$} \\
\hline & & 2 & 3 & & \\
\hline Rock type & $\mathrm{AP}$ & M & M & NS & I \\
\hline $\mathrm{SiO}_{2}$ & 39.45 & 37.19 & 36.38 & 38.49 & 39.85 \\
\hline $\mathrm{TiO}_{2}$ & 1.35 & 2.93 & 1.90 & 2.57 & 2.33 \\
\hline $\mathrm{Al}_{2} \mathrm{O}_{3}$ & 12.02 & 13.88 & 15.20 & 10.78 & 11.05 \\
\hline $\mathrm{Cr}_{2} \mathrm{O}_{3}$ & - & - & - & 0.03 & - \\
\hline $\mathrm{FeO}$ & 12.04 & 11.68 & 12.76 & 16.87 & 12.44 \\
\hline $\mathrm{MnO}$ & 0.48 & 0.42 & 0.43 & 0.42 & 0.51 \\
\hline $\mathrm{MgO}$ & 18.63 & 17.82 & 17.53 & 15.67 & 18.03 \\
\hline $\mathrm{CaO}$ & 0.01 & 0.04 & 0.03 & - & - \\
\hline $\mathrm{Na}_{2} \mathrm{O}$ & 0.32 & 0.44 & 0.39 & 0.28 & 0.12 \\
\hline $\mathrm{K}_{2} \mathrm{O}$ & 9.56 & 9.22 & 9.32 & 9.36 & 10.09 \\
\hline Total & 93.87 & 93.62 & 93.95 & 94.48 & 94.43 \\
\hline \multicolumn{6}{|l|}{$\mathrm{O}=24$} \\
\hline $\mathrm{Si}$ & 5.947 & 5.633 & 5.484 & 5.923 & 6.042 \\
\hline $\mathrm{Al}^{\mathrm{IV}}$ & 2.053 & 2.367 & 2.516 & 1.954 & 1.958 \\
\hline $\mathrm{Al}^{\mathrm{VI}}$ & 0.081 & 0.109 & 0.182 & - & 0.015 \\
\hline $\mathrm{Ti}$ & 0.153 & 0.334 & 0.216 & 0.297 & 0.266 \\
\hline $\mathrm{Fe}^{2}$ & 1.518 & 1.479 & 1.609 & 2.171 & 1.577 \\
\hline $\mathrm{Cr}$ & - & - & - & 0.004 & - \\
\hline $\mathrm{Mn}$ & 0.061 & 0.054 & 0.055 & 0.055 & 0.066 \\
\hline $\mathrm{Mg}$ & 4.187 & 4.024 & 3.939 & 3.595 & 4.075 \\
\hline $\mathrm{Ca}$ & 0.002 & 0.006 & 0.004 & 0.001 & - \\
\hline $\mathrm{Na}$ & 0.094 & 0.129 & 0.115 & 0.084 & 0.035 \\
\hline K & 1.839 & 1.781 & 1.792 & 1.838 & 1.952 \\
\hline Cations & 15.935 & 15.916 & 15.912 & 15.922 & 15.986 \\
\hline $\mathrm{Fe}_{-} \mathrm{FeMg}$ & 0.27 & 0.27 & 0.29 & 0.38 & 0.28 \\
\hline $\mathrm{Mg}_{-} \mathrm{FeMg}$ & 0.73 & 0.73 & 0.71 & 0.62 & 0.72 \\
\hline $\begin{array}{c}\mathrm{FeO} /(\mathrm{FeO}+ \\
\mathrm{MgO})\end{array}$ & 0.39 & 0.40 & 0.42 & 0.52 & 0.41 \\
\hline A.S.I. & 1.1 & 1.3 & 1.4 & 1.0 & 1.0 \\
\hline
\end{tabular}

AP: Alkali pyroxenite; M: Melteigite; NS: Nepheline syenite; I: Ijolite; A.S.I.: Alumina Saturation Index.

area. Electron probe micro analyses of opaque minerals from ijolite, melteigite and alkali pyroxenite have been presented in table 8 . The $\mathrm{FeO}^{(t)}$ content of analyzed opaque minerals range from 81.95 to $88.72 \mathrm{wt} \%$ with relatively lesser amounts of $\mathrm{TiO}_{2}$ (ranging from 3.46-5.68 wt\%). The analyzed chemical compositions of opaque minerals correspond to magnetite.

It is to be noted that the mineral-chemical data for differentiation centres (related to Sylhet Traps) of northeastern India were practically absent since long. However, very recently, Melluso et al (2010) published a splendid account of mineral compositions of Sung Valley ultramaficalkaline-carbonatite complex. In this view, a comparative assessment of mineralogical characters of Samchampi-Samteran alkaline complex (present study) and that of ultramafic-alkaline-carbonatite complex of Sung Valley, northeastern India will be
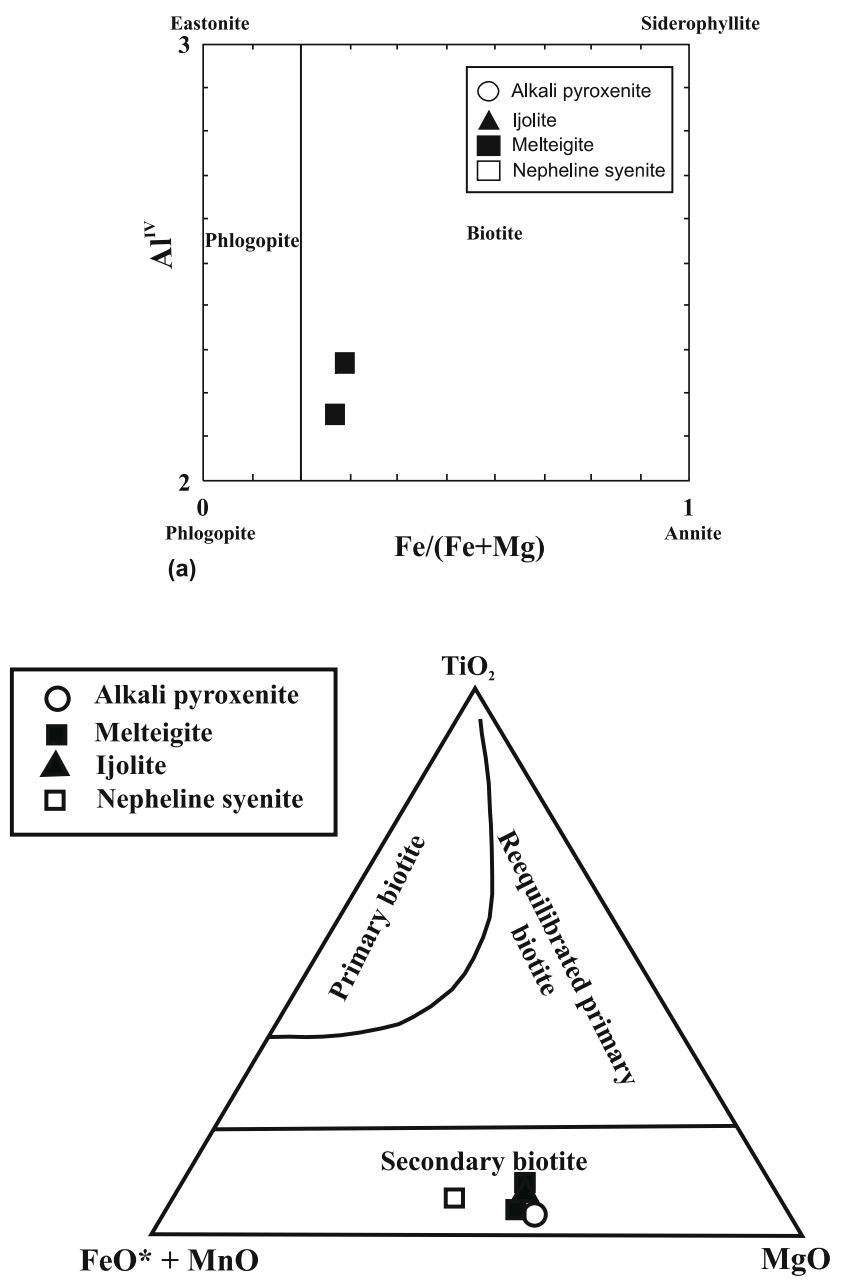

(b)

Figure 7. Plot of biotite compositions in eastonite-siderophyllite-phlogopite-annite diagram (Deer et al 1979, p. 212). Plot of biotite compositions in $\mathrm{TiO}_{2}-\mathrm{FeO}^{*}+\mathrm{MnO}-\mathrm{MgO}$ diagram (Nachit et al 2005).

worthwhile to present. The summary-table showing comparison of mineralogical characters between these two complexes has been given in table 9 .

\section{Geothermobarometry}

The relevant mineral chemical data obtained for constituent phases can be successfully applied for geothermobarometric estimations. Several thermometric and barometric methods (proposed by several workers) have been considered in the present study on the basis of available mineralogical compositions.

\subsection{Geothermometry}

\subsubsection{Pyroxene thermometry}

The electron microprobe analyses of pyroxene compositions are available for syenite, alkali pyroxenite, 
Table 6. EPM analyses of garnet.

\begin{tabular}{|c|c|c|c|c|c|}
\hline \multirow{2}{*}{$\frac{\text { Sample }}{\text { Analysis }}$} & \multirow{2}{*}{$\frac{\text { SAJ } 17}{1}$} & \multicolumn{4}{|c|}{ SAJ $4 \mathrm{~A}$} \\
\hline & & 2 & 3 & 4 & 5 \\
\hline Rock type & M & $\mathrm{S}$ & $\mathrm{S}$ & $\mathrm{S}$ & $\mathrm{S}$ \\
\hline $\mathrm{SiO}_{2}$ & 28.03 & 32.64 & 33.79 & 32.09 & 32.29 \\
\hline $\mathrm{TiO}_{2}$ & 12.88 & 3.62 & 4.06 & 7.10 & 6.66 \\
\hline $\mathrm{Al}_{2} \mathrm{O}_{3}$ & 2.67 & 1.31 & 1.55 & 0.67 & 0.71 \\
\hline $\mathrm{Cr}_{2} \mathrm{O}_{3}$ & 0.07 & 0.08 & 0.04 & 0.10 & 0.08 \\
\hline $\mathrm{FeO}$ & 0.90 & - & 0.67 & 1.83 & 1.63 \\
\hline $\mathrm{Fe}_{2} \mathrm{O}_{3}$ & 20.37 & 27.62 & 26.22 & 24.83 & 25.37 \\
\hline $\mathrm{MnO}$ & 0.60 & 0.60 & 0.54 & 0.55 & 0.58 \\
\hline $\mathrm{MgO}$ & 1.15 & 0.15 & 0.21 & 0.24 & 0.21 \\
\hline $\mathrm{CaO}$ & 31.91 & 32.08 & 32.21 & 31.53 & 31.52 \\
\hline $\mathrm{Na}_{2} \mathrm{O}$ & 0.14 & 0.21 & 0.26 & 0.33 & 0.35 \\
\hline Total & 98.71 & 98.31 & 99.55 & 99.27 & 99.40 \\
\hline \multicolumn{6}{|l|}{$\mathrm{O}=24$} \\
\hline TSi & 4.788 & 5.601 & 5.706 & 5.477 & 5.503 \\
\hline TAl & 0.536 & 0.264 & 0.294 & 0.134 & 0.142 \\
\hline Sum_ $_{-} \mathrm{T}$ & 5.325 & 5.865 & 6 & 5.611 & 5.645 \\
\hline $\mathrm{Al}^{\mathrm{VI}}$ & - & - & 0.014 & - & - \\
\hline $\mathrm{Fe}^{3}$ & 2.616 & 3.563 & 3.328 & 3.186 & 3.25 \\
\hline $\mathrm{Ti}$ & 1.655 & 0.467 & 0.516 & 0.912 & 0.854 \\
\hline $\mathrm{Cr}$ & 0.009 & 0.011 & 0.006 & 0.013 & 0.01 \\
\hline $\mathrm{Sum}_{-} \mathrm{A}$ & 4 & 4 & 4 & 4 & 4 \\
\hline $\mathrm{Fe}^{2}$ & 0.129 & - & 0.094 & 0.261 & 0.232 \\
\hline $\mathrm{Mg}$ & 0.292 & 0.039 & 0.052 & 0.061 & 0.054 \\
\hline $\mathrm{Mn}$ & 0.086 & 0.087 & 0.078 & 0.079 & 0.084 \\
\hline $\mathrm{Ca}$ & 5.840 & 5.898 & 5.827 & 5.766 & 5.755 \\
\hline $\mathrm{Na}$ & 0.048 & 0.070 & 0.085 & 0.111 & 0.116 \\
\hline Sum_B & 6.396 & 6.094 & 6.136 & 6.278 & 6.241 \\
\hline Sum_cat & 16 & 16 & 16 & 16 & 16 \\
\hline And & 82.75 & 95.52 & 91.38 & 95.58 & 95.51 \\
\hline Gross & 9.01 & 1.94 & 6.10 & 1.22 & 1.48 \\
\hline Pyrope & 6.15 & 0.69 & 0.95 & 1.22 & 1.06 \\
\hline Spess & 1.82 & 1.55 & 1.42 & 1.59 & 1.64 \\
\hline Uvaro & 0.28 & 0.30 & 0.16 & 0.39 & 0.31 \\
\hline XCagnt & 0.920 & 0.979 & 0.963 & 0.935 & 0.940 \\
\hline XFegnt & 0.020 & - & 0.016 & 0.042 & 0.038 \\
\hline XMggnt & 0.046 & 0.006 & 0.009 & 0.010 & 0.009 \\
\hline Fe_Mggnt & 0.442 & - & 1.808 & 4.279 & 4.296 \\
\hline
\end{tabular}

M: Melteigite; S: Syenite.

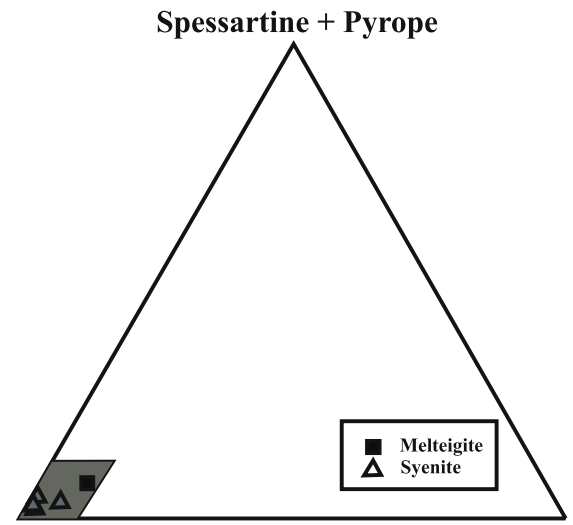

Ti- Andradite

Grossular

Figure 8. Compositional range of Samchampi-Samteran garnets (shaded area) in Spessartine + Pyrope - TiAndradite-Grossular system (Dingwell and Brearley 1985).
Table 7. EPM analyses of sphene.

\begin{tabular}{lcccc}
\hline Sample & SAJ 11 & SAJ 19 & SAJ 4B & SAJ 4A \\
\hline Analysis & 1 & 2 & 3 & 4 \\
Rock type & $\mathrm{AP}$ & $\mathrm{M}$ & $\mathrm{AG}$ & $\mathrm{S}$ \\
$\mathrm{SiO}_{2}$ & 29.57 & 29.70 & 29.81 & 29.51 \\
$\mathrm{TiO}_{2}$ & 34.85 & 33.45 & 37.27 & 35.06 \\
$\mathrm{Al}_{2} \mathrm{O}_{3}$ & 1.09 & 0.61 & 0.40 & 1.33 \\
$\mathrm{Cr}_{2} \mathrm{O}_{3}$ & 0.10 & 0.06 & 0.03 & 0.09 \\
$\mathrm{FeO}$ & 1.67 & 2.47 & 1.72 & 2.21 \\
$\mathrm{MnO}$ & 0.02 & 0.07 & 0.06 & 0.05 \\
$\mathrm{MgO}$ & 0.05 & 0.05 & 0.03 & - \\
$\mathrm{CaO}$ & 26.62 & 26.19 & 26.90 & 27.81 \\
$\mathrm{Na} 2 \mathrm{O}$ & 0.36 & 0.15 & 0.23 & 0.11 \\
$\mathrm{Total}$ & 94.34 & 92.74 & 96.45 & 96.17 \\
$\mathrm{O}=20$ & & & & \\
$\mathrm{Si}$ & 4.108 & 4.205 & 4.042 & 4.056 \\
$\mathrm{Al}$ & 0.178 & 0.102 & 0.215 & 0.064 \\
$\mathrm{Ti}$ & 3.642 & 3.562 & 3.612 & 3.814 \\
$\mathrm{Cr}$ & 0.010 & 0.007 & 0.010 & 0.003 \\
$\mathrm{Mg}$ & 0.010 & 0.010 & - & 0.007 \\
$\mathrm{Fe}$ & 0.194 & 0.292 & 0.253 & 0.195 \\
$\mathrm{Mn}$ & 0.002 & 0.008 & 0.006 & 0.007 \\
$\mathrm{Na}$ & 0.097 & 0.040 & 0.030 & 0.061 \\
$\mathrm{Ca}$ & 3.962 & 3.973 & 4.081 & 3.921 \\
$\mathrm{Sum}$ cats & 12.203 & 12.199 & 12.249 & 12.128 \\
\hline & & & &
\end{tabular}

AP: Alkali pyroxenite; M: Melteigite; AG: Alkali gabbro; S: Syenite.

Table 8. EPM analyses of opaque oxide.

\begin{tabular}{|c|c|c|c|}
\hline Sample & SAJ 17 & SAJ 11 & SAJ : \\
\hline Analysis & 1 & 2 & 3 \\
\hline Rock type & $\mathrm{M}$ & $\mathrm{AP}$ & I \\
\hline $\mathrm{SiO}_{2}$ & 0.05 & 0.01 & 0.05 \\
\hline $\mathrm{TiO}_{2}$ & 5.68 & 4.92 & 3.46 \\
\hline $\mathrm{Al}_{2} \mathrm{O}_{3}$ & 0.90 & 0.82 & 0.51 \\
\hline $\mathrm{Cr}_{2} \mathrm{O}_{3}$ & 0.06 & 0.03 & - \\
\hline $\mathrm{FeO}^{(\mathrm{t})}$ & 81.95 & 83.83 & 88.72 \\
\hline $\mathrm{MnO}$ & 1.84 & 2.17 & 0.40 \\
\hline $\mathrm{MgO}$ & 0.66 & 0.67 & 0.0 \\
\hline $\mathrm{CaO}$ & - & 0.01 & - \\
\hline $\mathrm{NiO}$ & 0.05 & 0.07 & 0.02 \\
\hline $\mathrm{ZnO}$ & 0.23 & 0.31 & 0.09 \\
\hline Total & 100.32 & 98.62 & 99.41 \\
\hline \multicolumn{4}{|l|}{$\mathrm{O}=4$} \\
\hline $\mathrm{Si}$ & 0.0013 & 0.0002 & 0.0021 \\
\hline $\mathrm{Ti}$ & 0.1114 & 0.1425 & 0.1002 \\
\hline $\mathrm{Al}$ & 0.0278 & 0.0371 & 0.0230 \\
\hline $\mathrm{Cr}$ & 0.0013 & 0.0010 & - \\
\hline $\mathrm{Fe}^{3+}$ & 1.7455 & 1.6764 & 1.7724 \\
\hline $\mathrm{Fe}^{2+}$ & 0.0409 & 1.0228 & 1.0842 \\
\hline Mn & 0.0406 & 0.0708 & 0.0132 \\
\hline $\mathrm{Mg}$ & 0.0255 & 0.0382 & 0.0016 \\
\hline $\mathrm{Ca}$ & - & 0.0005 & 0.0002 \\
\hline $\mathrm{Ni}$ & 0.0011 & 0.0021 & 0.0006 \\
\hline $\mathrm{Zn}$ & 0.0045 & 0.0088 & 0.0027 \\
\hline CatTot & 2 & 3 & 3 \\
\hline $\mathrm{X}$ & 27.56 & 15.54 & 56.85 \\
\hline $\mathrm{Y}$ & 16.12 & 12.48 & 42.67 \\
\hline
\end{tabular}

M: Melteigite; AP: Alkali pyroxenite; I: Ijolite. 


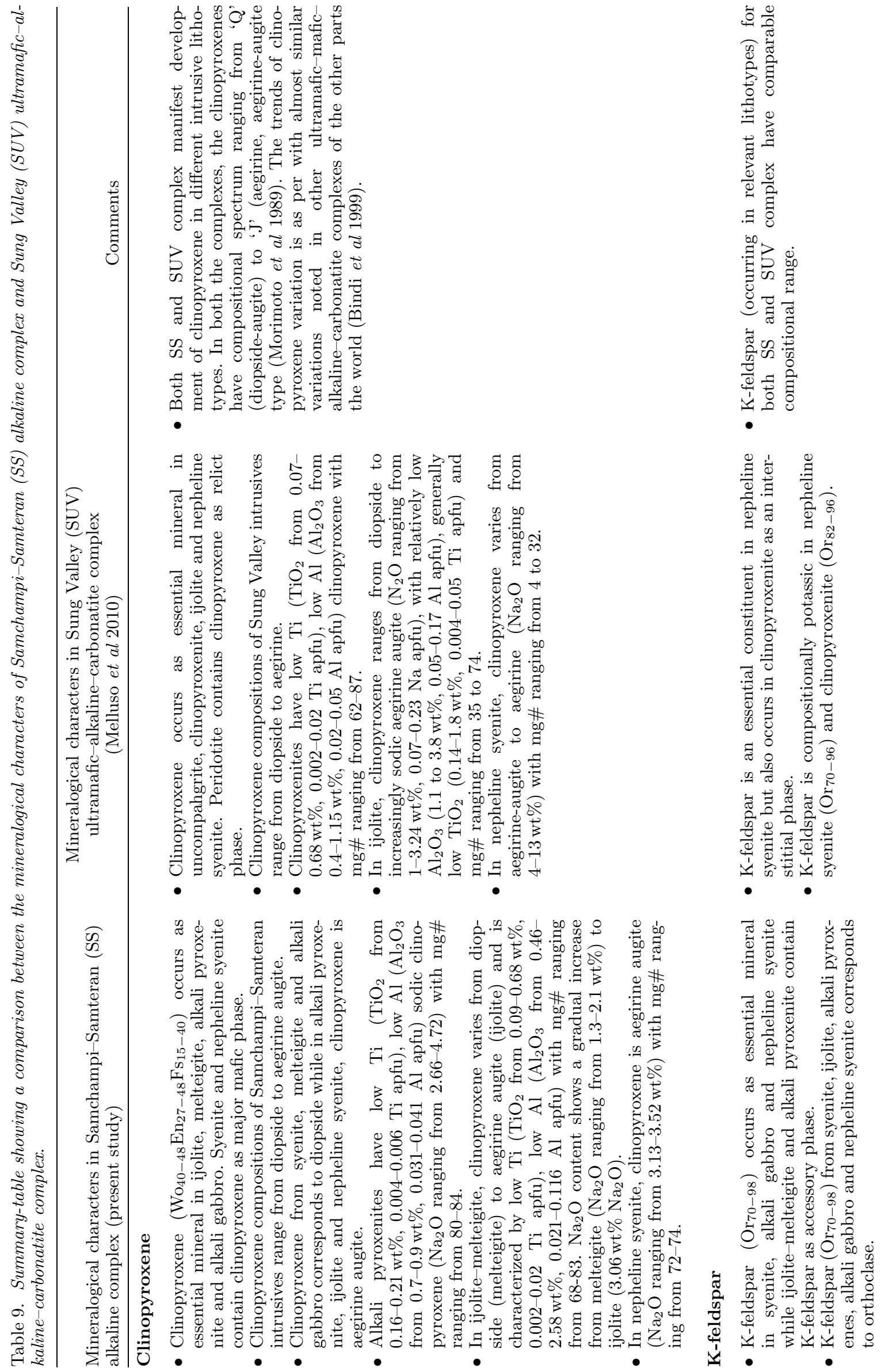



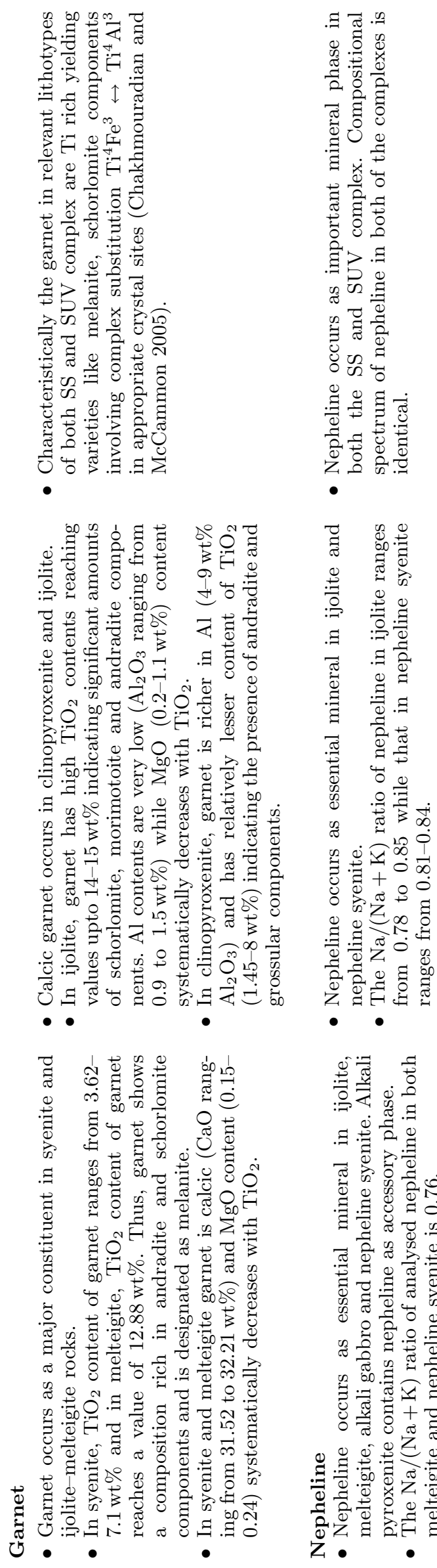

alkali gabbro, ijolite-meleigite and nepheline syenite. Out of these, the analyzed pyroxene compositions corresponding to syenite, melteigite and alkali gabbro have been classified as 'Quad' pyroxenes (as mentioned earlier). Therefore, in order to determine the temperature, pyroxene compositions can be assessed in terms of pyroxene thermometry (Lindsley 1983), in order to determine temperature. The procedure involves simple recalculation of the analyzed pyroxene compositions in terms of several end-member molecules and subsequent plotting of those recalculated molecules in terms of experimentally determined pyroxene thermometer contours. The investigated pyroxene compositions when projected into Lindsley's diagram (figure 9), reveal that the syenite represents a lower temperature of equilibration at $\sim 400^{\circ} \mathrm{C}$ while, melteigite and alkali gabbro record a close range of temperature of equilibration ranging from $\sim 450^{\circ}-600^{\circ} \mathrm{C}$.

\subsubsection{Clinopyroxene-garnet thermometry}

Geothermometric estimations have also been evaluated on the basis of coexisting clinopyroxenegarnet compositions for syenite and ijolitemelteigite rocks following the methods proposed by Mysen and Heier (1972); Raheim and Green (1974); Mori and Green (1978); Krogh (1988); Pattison and Newton (1989); Sengupta et al (1989) and Ai (1994). Temperatures calculated for syenite and ijolite-melteigite from different thermometric methods have been furnished in table 9 . The average temperature deduced for syenite ranges from $569^{\circ}-618^{\circ} \mathrm{C}$ (table 10 ). The ijolite-melteigite suite of rocks exhibits a relatively higher range of equilibration temperature ranging from $687^{\circ}$ to $1373^{\circ} \mathrm{C}$ (table 10).

\subsubsection{Two feldspar thermometry}

Two feldspar thermometry with available electron microprobe data for syenite was estimated following the method of Haselton et al (1983). The calculated two feldspar thermometry yields an average equilibration temperature of $583^{\circ} \mathrm{C}$ for syenite (table 10).

\subsection{Geobarometry}

\subsubsection{Pyroxene barometry}

Ashchepkov (2009) put forward reliable barometric methods based on pyroxene compositions. In the present study, geobarometric estimates involve analyzed pyroxene compositions from syenite, ijolite-melteigite, alkali pyroxenite, alkali gabbro and nepheline syenite. The deduced values 


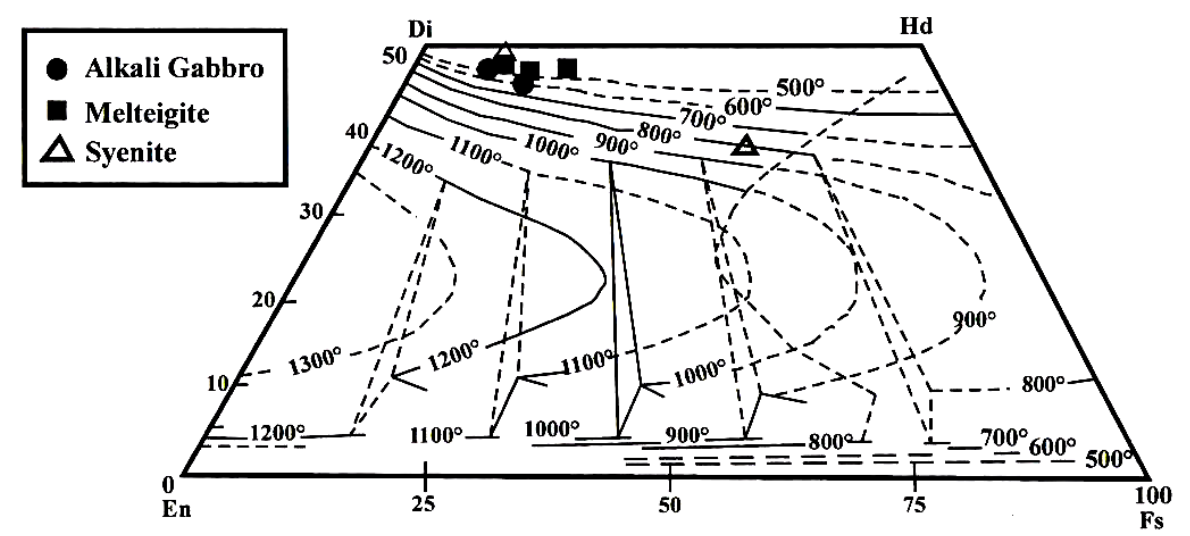

Figure 9. Recalculated pyroxene compositions plotted in terms of Di-Hd-En-Fs diagram for thermometry (Lindsley 1983).

Table 10. Geothermometric data of different lithotypes.

\begin{tabular}{llcc}
\hline Rock type & \multicolumn{1}{c}{ Thermometric method } & $\begin{array}{c}\text { Temperature } \\
\left({ }^{\circ} \mathrm{C}\right)\end{array}$ & $\begin{array}{c}\text { Average } \\
\text { temperature }\left({ }^{\circ} \mathrm{C}\right)\end{array}$ \\
\hline Melteigite-ijolite & $\begin{array}{l}\text { Cpx-Garnet chemistry } \\
\text { (Raheim and Green 1974) }\end{array}$ & 1373.8 & 1373.8 \\
& $\begin{array}{l}\text { Cpx-Garnet chemistry } \\
\text { (Sengupta } \text { et al 1989) }\end{array}$ & 687.0 & 687.0 \\
& $\begin{array}{l}\text { Cpx-Garnet chemistry } \\
\text { (Ai 1994) }\end{array}$ & 942.2 & 942.2 \\
Syenite & $\begin{array}{l}\text { Cpx-Garnet chemistry } \\
\text { (Raheim and Green 1974) }\end{array}$ & 569.1 & 569.1 \\
& $\begin{array}{l}\text { Cpx-Garnet chemistry } \\
\text { (Krogh 1988) }\end{array}$ & 484.3 & 684.3 \\
& $\begin{array}{l}\text { Cpx-Garnet chemistry } \\
\text { (Pattison and Newton 1989) }\end{array}$ & 617.7 & 581.8 \\
& $\begin{array}{l}\text { Cpx-Garnet chemistry } \\
\text { (Mysen and Heier 1972) }\end{array}$ & 581.8 & 574.4 \\
& $\begin{array}{l}\text { Cpx-Garnet chemistry } \\
\text { (Mori and Green 1978) } \\
\text { Two feldspar thermometry } \\
\text { (Haselton et al 1983) }\end{array}$ & 584.4 & 583.3 \\
\hline
\end{tabular}

(table 11) reveal a closely comparable pressure for ijolite-melteigite, alkali pyroxenite, alkali gabbro, and nepheline syenite (average pressure values ranging from $5.32-7.72 \mathrm{~kb}$ ) corresponding to a depth of crystallization of $\sim 16-23 \mathrm{~km}$. Syenite exhibits a pressure of $11.81 \mathrm{~kb}$ (table 11) indicating a deeper level of intrusion at about $33 \mathrm{~km}$ (assuming $3 \mathrm{~km} / \mathrm{kb}$ ).

\subsubsection{Clinopyroxene-plagioclase barometry}

Barometric estimates involving coexisting clinopyroxene-plagioclase compositions have been adopted for syenite following the method proposed by Ellis and Green (1979). The calculated pressure of $11.61 \mathrm{~kb}$ corresponds to a depth of intrusion of $\sim 33 \mathrm{~km}$.
Table 11. Geobarometric data of different intrusives.

\begin{tabular}{llcr}
\hline Rock type & Barometric method & P in kb & $\begin{array}{c}\text { Avg. P } \\
\text { in kb }\end{array}$ \\
\hline Alkali pyroxenite & $\begin{array}{l}\text { Cpx chemistry } \\
\text { (Ashchepkov 2009) }\end{array}$ & 7.72 & 7.72 \\
Melteigite-ijolite & & \\
& $\begin{array}{l}\text { Cpx chemistry } \\
\text { (Ashchepkov 2009) }\end{array}$ & 7.1 & \\
& & 6.58 & 6.68 \\
Alkali gabbro & Cpx chemistry & 4.93 & 5.32 \\
Syenite & (Ashchepkov 2009) & 5.71 & \\
& Cpx chemistry & 11.81 & 11.81 \\
& (Ashchepkov 2009) & & \\
& Cpx-Garnet & 11.61 & 11.71 \\
& $\quad$ chemistry & & \\
& (Ellis and Green & & \\
$\quad$ 1979) & 4.93 & 5.32 \\
Nepheline syenite & Cpx chemistry & & \\
& (Ashchepkov 2009) & 5.71 & \\
\hline
\end{tabular}


Table 12. Whole rock major oxide data (wt.\%).

\begin{tabular}{|c|c|c|c|c|c|c|c|c|c|c|c|}
\hline & AP1* & $\mathrm{AP} 2^{* *}$ & $\mathrm{AP}^{* *}$ & $\mathrm{I}^{*}$ & $\mathrm{I} 2^{* *}$ & $\mathrm{M}^{*}$ & $\mathrm{M} 2^{* *}$ & $\mathrm{~S}^{*}$ & $\mathrm{NS}^{*}$ & $\mathrm{NS}^{* *}$ & $\mathrm{C}^{*}$ \\
\hline $\mathrm{SiO}_{2}$ & 44.09 & 44.07 & 46.26 & 43.63 & 38.50 & 45.20 & 38.09 & 53.66 & 52.48 & 57.07 & 5.48 \\
\hline $\mathrm{TiO}_{2}$ & 1.00 & 0.66 & 0.26 & 1.25 & 1.25 & 0.90 & 0.87 & 1.27 & 0.98 & 0.69 & 0.46 \\
\hline $\mathrm{Al}_{2} \mathrm{O}_{3}$ & 14.90 & 9.27 & 6.52 & 13.45 & 11.84 & 3.20 & 7.83 & 9.12 & 16.80 & 14.43 & 0.50 \\
\hline $\mathrm{Fe}_{2} \mathrm{O}_{3}$ & 10.89 & 3.66 & 5.93 & 5.38 & 3.83 & 5.80 & 2.36 & 10.06 & 3.65 & 2.35 & 3.92 \\
\hline $\mathrm{FeO}$ & 0.70 & 3.58 & 3.36 & 2.75 & 6.28 & 5.91 & 2.00 & 3.27 & 3.67 & 1.14 & 2.41 \\
\hline $\mathrm{MnO}$ & 0.12 & 0.29 & 0.82 & 0.11 & 0.31 & 0.38 & 0.27 & 0.05 & 0.11 & 0.02 & 0.20 \\
\hline $\mathrm{MgO}$ & 4.48 & 5.33 & 7.31 & 8.04 & 10.36 & 14.80 & 6.71 & 3.47 & 4.11 & 0.01 & 5.63 \\
\hline $\mathrm{CaO}$ & 20.27 & 16.30 & 17.61 & 11.33 & 9.45 & 16.58 & 18.17 & 13.02 & 6.07 & 0.10 & 46.32 \\
\hline $\mathrm{Na}_{2} \mathrm{O}$ & 1.93 & 6.50 & 5.25 & 4.35 & 4.67 & 1.62 & 5.87 & 2.03 & 4.48 & 9.25 & 0.13 \\
\hline $\mathrm{K}_{2} \mathrm{O}$ & 2.05 & 2.87 & 1.00 & 4.21 & 3.71 & 2.51 & 0.62 & 2.13 & 4.66 & 11.75 & 0.16 \\
\hline $\mathrm{P}_{2} \mathrm{O}_{5}$ & 0.10 & 1.74 & 2.30 & 0.40 & 1.48 & 0.62 & 3.89 & 0.10 & 0.17 & 0.15 & 0.90 \\
\hline $\mathrm{CO}_{2}$ & n.d & - & - & 1.84 & - & 0.84 & - & - & n.d & - & 31.45 \\
\hline LOI & - & 5.52 & 1.18 & - & 6.12 & - & 8.87 & - & 1.63 & 0.88 & - \\
\hline Total & 100.53 & 99.79 & 97.80 & 96.74 & 97.80 & 98.36 & 95.55 & 98.18 & 98.81 & 97.84 & 97.56 \\
\hline \multicolumn{12}{|l|}{ CIPW norm } \\
\hline Quartz & - & - & - & - & - & - & - & 12.67 & - & - & - \\
\hline Plagioclase & 25.94 & - & - & 4.74 & 0.39 & - & - & 26.66 & 37.46 & - & 0.31 \\
\hline Orthoclase & 6.14 & - & 1.28 & 20.18 & - & - & - & 12.59 & 27.54 & 65.91 & - \\
\hline Nepheline & 8.85 & 17.17 & 15.15 & 19.94 & 21.41 & 1.35 & 19.95 & - & 6.73 & 4.77 & 0.60 \\
\hline Leucite & 4.68 & 13.30 & 3.63 & 3.69 & 17.19 & 11.63 & 2.87 & - & - & 2.76 & - \\
\hline Kalsilite & - & - & - & - & - & - & - & - & - & - & 0.54 \\
\hline Diopside & 24.07 & 36.77 & 51.51 & 28.97 & 24.18 & 45.38 & 41.20 & 18.64 & 13.54 & - & - \\
\hline Wollastonite & 17.85 & - & 3.41 & - & - & - & 0.27 & 12.74 & - & - & - \\
\hline Olivine & - & 1.12 & - & 4.62 & 15.15 & 16.72 & - & - & 4.37 & 0.78 & 10.44 \\
\hline Larnite & - & 7.42 & - & - & 2.02 & 4.88 & 3.71 & - & - & - & 7.67 \\
\hline Acmite & - & 10.59 & 14.50 & - & - & 9.89 & 6.83 & - & - & 6.80 & - \\
\hline $\mathrm{Na}_{2} \mathrm{SiO}_{3}$ & - & 2.63 & - & - & - & - & 1.19 & - & - & 14.37 & - \\
\hline Ilmenite & 1.74 & 1.25 & 0.49 & 2.37 & 2.37 & 1.71 & 1.65 & 2.41 & 1.86 & 1.31 & 0.87 \\
\hline Magnetite & - & - & 1.33 & 5.60 & 5.55 & 3.45 & - & 7.02 & 5.29 & - & 5.68 \\
\hline Hematite & 10.89 & - & - & 1.52 & - & - & - & 5.22 & - & - & - \\
\hline Apatite & 0.23 & 4.03 & 5.33 & 0.93 & 3.43 & 1.44 & 9.01 & 0.23 & 0.39 & 0.35 & 2.09 \\
\hline Perovskite & 0.15 & - & - & - & - & - & - & - & - & - & - \\
\hline Calcite & - & - & - & 4.18 & - & 1.91 & - & - & - & - & 71.53 \\
\hline D.I. & 45.60 & 30.50 & 20.10 & 48.60 & 39.00 & 13.00 & 22.80 & 51.90 & 71.70 & 73.40 & 1.50 \\
\hline A.I. & 0.36 & 1.50 & 1.50 & 0.87 & 0.98 & 1.70 & 1.32 & 0.63 & 0.74 & 1.94 & 0.82 \\
\hline
\end{tabular}

AP: Alkali pyroxenite; I: Ijolite; M: Melteigite; S: Syenite; NS: Nepheline syenite; and C: Carbonanite.

*Analyses taken from Nag et al (1999).

**Analyses taken from Kumar et al (1989).

\section{Petrochemistry}

Whole rock major oxide data for alkali pyroxenite, ijolite, melteigite, syenite, nepheline syenite and carbonatite from Samchampi-Samteran alkaline complex (after Kumar et al 1989; Nag et al 1999) and the corresponding CIPW norms have been furnished in table 12. The alkali pyroxenite (AP1, AP2 and AP3) and ijolite-melteigite (I1, I2, M1 and M2) rocks are characterized by alkaline normative minerals like nepheline, leucite and larnite. CIPW normative compositions suggest the alkaline nature of these rocks. The agpaitic indices [A.I. $=\left(\mathrm{NaO}+\mathrm{K}_{2} \mathrm{O}\right) / \mathrm{Al}_{2} \mathrm{O}_{3}$ ] for two alkali pyroxenite samples (AP2 and AP3) are $>1$ suggesting their agpaitic nature while one alkali pyroxenite sample (AP1) is miaskitic with A.I $=0.36$. The ijolite samples (I1 and I2) are miaskitic and the melteigite samples (M1 and M2) are agpaitic in nature. The miaskitic affinity of a nepheline syenite sample (NS1) reflects a close alkaline-carbonatite association (Srivastava and Sinha 2007). Major oxide variations of the Samchampi-Samteran rocks are plotted against differentiation index (D.I.) in Harker variation diagrams (figure 10). Differentiation Index (D.I.) has been calculated as normative $\mathrm{Qtz}+\mathrm{Or}+\mathrm{Ab}+\mathrm{Ne}+\mathrm{Lc}$ (after Thornton and Tuttle 1960). Figure 10 shows that $\mathrm{SiO}_{2}$, $\mathrm{Al}_{2} \mathrm{O}_{3}$ and total alkalies $\left(\mathrm{NaO}+\mathrm{K}_{2} \mathrm{O}\right)$ gradually increase with rising D.I. while $\mathrm{TiO}_{2}, \mathrm{FeO}^{(\mathrm{t})}, \mathrm{CaO}$ 

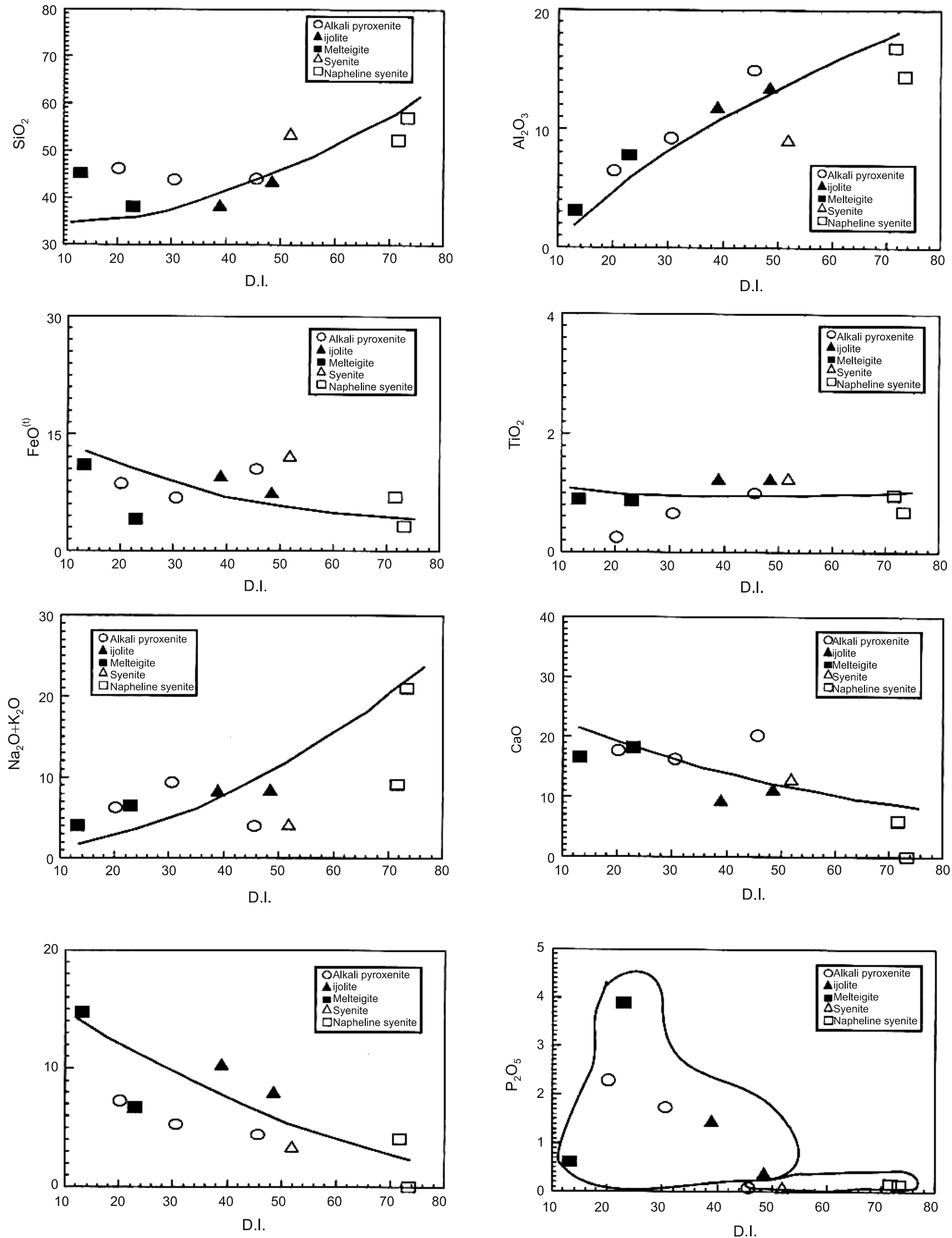

Figure 10. D.I. vs. major oxides variation diagram for Samchampi-Samteran rocks. 


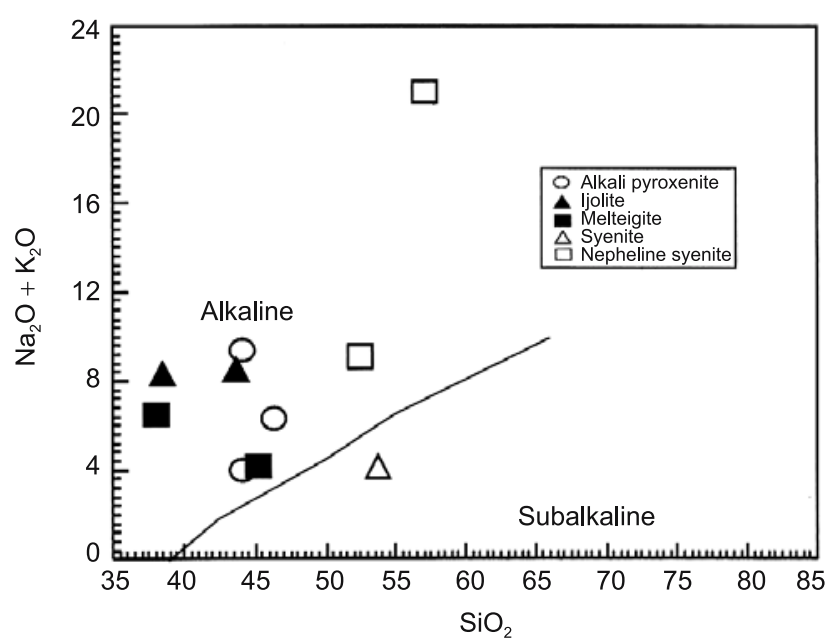

Figure 11. Total alkali vs. silica diagram for SamchampiSamteran rocks. Field boundary after Macdonald and Katsura (1964).

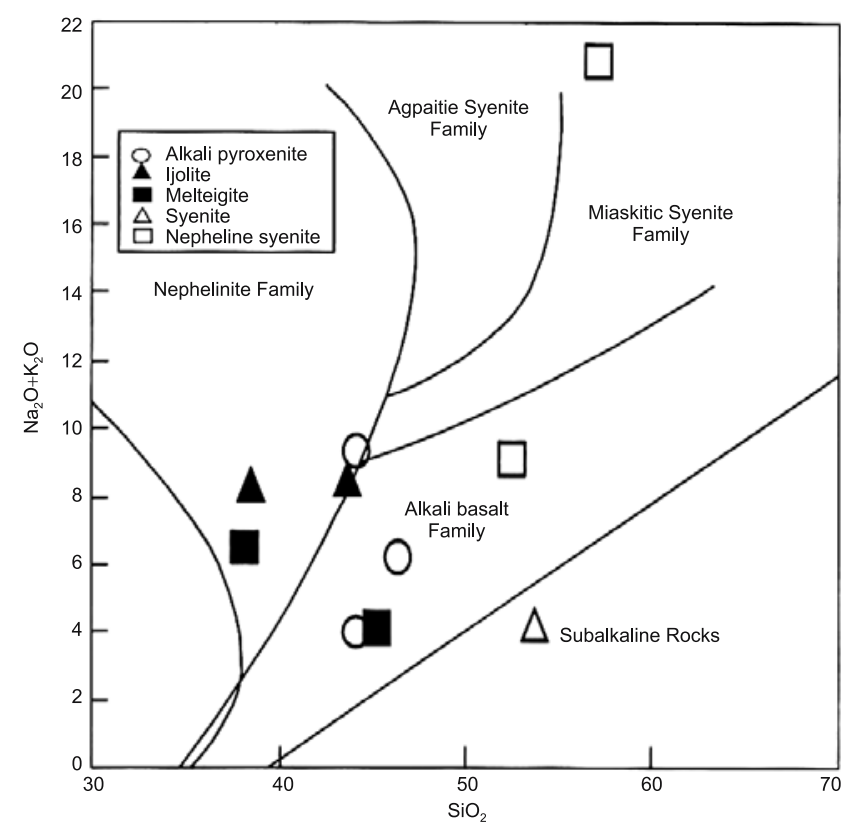

Figure 12. Total alkali vs. silica diagram for SamchampiSamteran rocks. Field boundaries after Hyndaman (1985).

and $\mathrm{MgO}$ systematically fall with increasing D.I. The general trend of major oxide variations for the Samchampi-Samteran rocks is very much consistent with normal magmatic differentiation. But the variation of $\mathrm{P}_{2} \mathrm{O}_{5}$ with respect to D.I. in alkaline ultramafic (alkali pyroxenite) and alkaline mafic (ijolite-melteigite) rocks is distinctly anomalous. Phosphorous, being a high charge density (HCD) cation is normally concentrated in late stage differentiates, i.e., in more silicic rocks. However, for the Samchampi-Samteran complex, the alkaline ultramafic and alkaline mafic rocks are more enriched in $\mathrm{P}_{2} \mathrm{O}_{5}$ than their felsic counterparts (syenite, nepheline syenite) (figure 10). The geochemical characters of the Samchampi-Samteran rocks are further illustrated in figures 11 and 12. In the total alkali vs. silica diagram (figure 11), the Samchampi-Samteran samples distinctly occupy the 'alkaline' field with a lone plot of syenite falling the 'subalkaline' field. Figure 12 depicts total alkali vs. silica variation diagram wherein two alkali pyroxenite samples plot in the Alkali basalt family and one sample falls in the Nephelinite family. The ijolite samples together with one melteigite sample correspond to the Nephelinite family while one melteigite sample belongs to the Alkali basalt family. Nepheline syenite occupies the fields of Alkali basalt family and Miaskitic syenite family while the syenite sample plots in the field of subalkaline rocks. Compositional plots of Samchampi-Samteran samples in the Petrogeny's Residua System (figure 13) reveal that only the syenite sample falls in the silica oversaturated field. Plots for alkali pyroxenite and ijolite fall in the nepheline field while melteigite and nepheline syenite samples show compositional occupancy in both nepheline and leucite fields. However, characteristically the samples falling within leucite field are devoid of normative and modal leucite. These observations suggest that the investigated rocks crystallized in a high $\mathrm{P}_{\mathrm{H} 2 \mathrm{O}}$ regime where the stability field of leucite gradually contracted and ceased to exist under the influence of a water enriched milieu. Presence of amphibole in syenite and biotite in the alkaline felsic and alkaline maficultramafic rocks of Samchampi-Samteran also supports the role of a hydrous environment in the formation of Samchampi-Samteran rocks. $\mathrm{CaO}$ vs. $\left(\mathrm{Na}_{2} \mathrm{O}+\mathrm{K}_{2} \mathrm{O}\right)$ variations in Samchampi-Samteran rocks depict a trend which corroborates the antipathetic relationship between $\mathrm{CaO}$ and total alkalies in a narrow dispersion zone characteristic of silicaundersaturated plutons (figure 14). Similar trend is observed for two other well-studied alkaline complexes of northeastern India, viz., Sung Valley and Jasra (figure 14). Thus a geochemical association of the Samchampi-Samteran rocks with Sung Valley and Jasra alkaline complexes is documented.

\section{Discussion}

Lineament controlled emplacement comprising ultramafic-mafic-alkaline-carbonatite complexes are often associated with rift tectonics (Woolley 1989). In northeastern India, such lineament controlled differentiated complexes are relegated to an incubating mantle plume and concomitant extrusion of Bengal-Rajmahal-Sylhet volcanics (Evans 1964; Gupta and Sen 1988; Nandy 1980, 2001). The presently investigated Samchampi-Samteran complex represents an excellent development of 


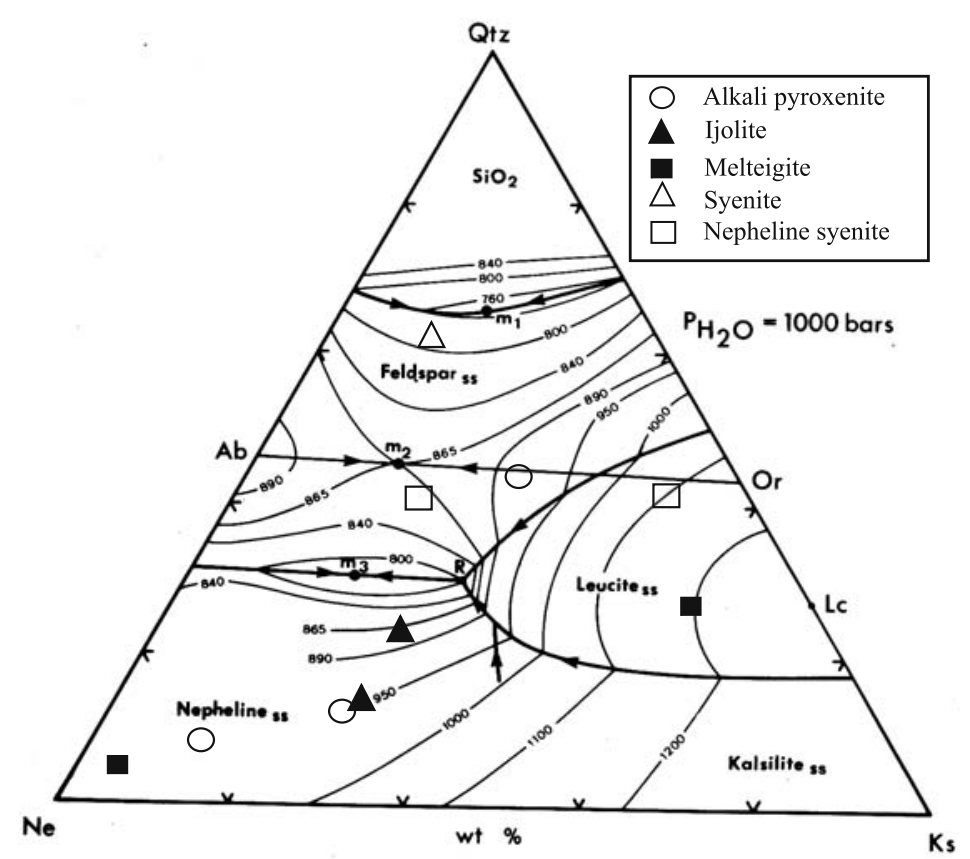

Figure 13. Compositional plots of Samchampi-Samteran rocks in Petrogeny's Residua System (after Hamilton and MacKnzie 1965).

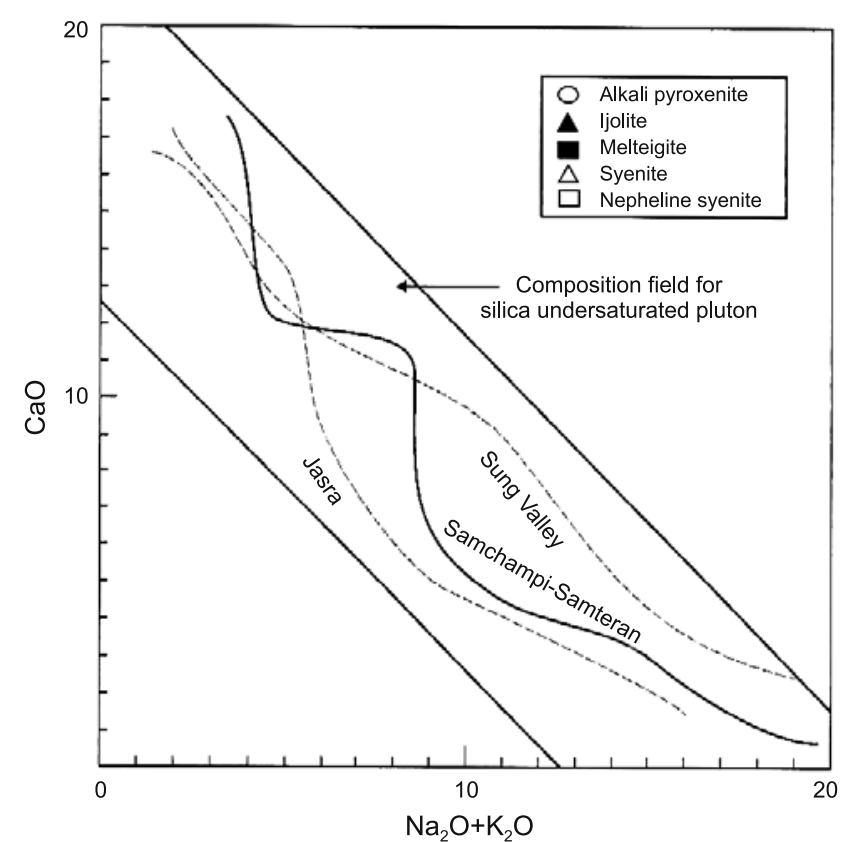

Figure 14. $\mathrm{CaO}$ vs. $\left(\mathrm{Na}_{2} \mathrm{O}+\mathrm{K}_{2} \mathrm{O}\right)$ diagram for SamchampiSamteran rocks (after Ratnakar and Leelanandam 1989).

such flood-basalt related differentiation centre where plug-like body of syenite has been intruded by ijolite-melteigite suite of rocks with occasional presence of alkali pyroxenite and alkali gabbro inliers. The pulsating magmatic activity was further manifested in terms of development of vanadium bearing titaniferous magnetite ore bodies and later intrusions of carbonatite and nepheline syenite dykes.
In the following paragraphs, we discuss few important points which would be meaningful to constrain petrogenesis of Samchampi-Samteran alkaline complex.

\subsection{Implications from mineral chemistry}

Chemical compositions of coexisting phases have been used for thermobarometric estimations. Critical assessment of pyroxene thermometry data suggests an equilibration temperature from $\sim 450^{\circ}-600^{\circ} \mathrm{C}$ for melteigites and alkali gabbro and $\sim 400^{\circ} \mathrm{C}$ for syenite. Other thermometric methods reveal a temperature of equilibration of $\sim 700^{\circ}-1373^{\circ} \mathrm{C}$ for ijolite-melteigite suite of rocks in contrast to relatively lower $\left(\sim 600^{\circ} \mathrm{C}\right)$ equilibration temperature for the syenites. These deduced temperatures for syenite speak in favour of subsolvus cooling. Barometric data based on pyroxene chemistry on the other hand critically deduce average pressure values from 5.32 to $7.72 \mathrm{~kb}$ for the ijolite-melteigite, alkali pyroxenite, alkali gabbro and nepheline syenite. The main syenite plug records an equilibration pressure of $\sim 11 \mathrm{~kb}$ indicating a deeper level of intrusion. It appears that the syenite equilibrated at a relatively greater depth than other intrusive variants of the SamchampiSamteran Complex.

\subsection{Role of metasomatism}

Petrographic observations reveal that two generations of clinopyroxene occur in ijolite-melteigite 


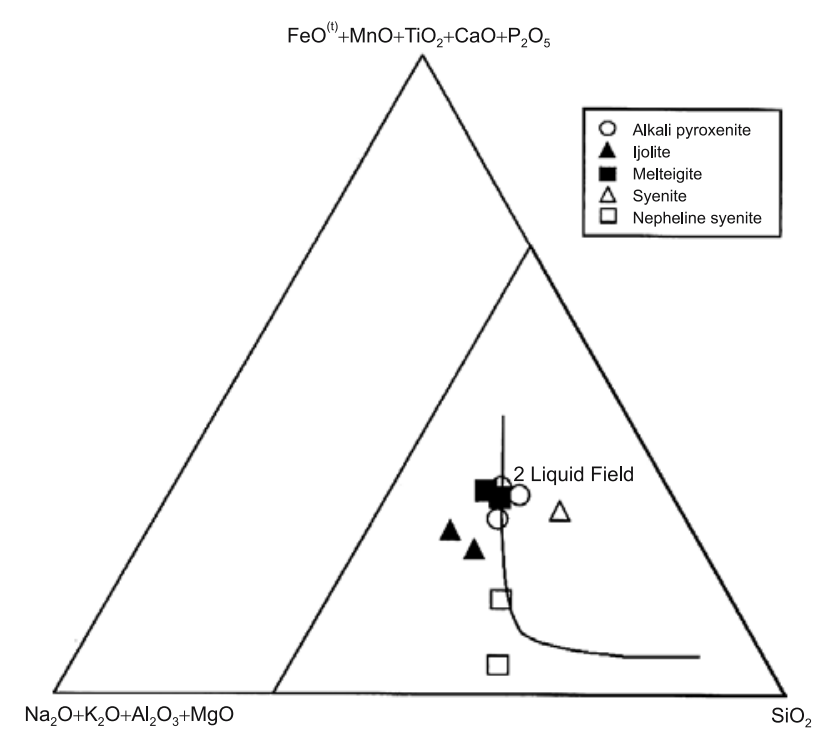

Figure 15. Compositional plots of Samchampi-Samteran rocks in Greig diagram (Philpotts 1982).

rocks. The earlier formed clinopyroxene occur as inclusions in nepheline (figure 4b) while the late formed clinopyroxene grains containing nepheline inclusions (figure 4c) are more sodic and have developed due to metasomatism. Vermicular intergrowths of nepheline within K-feldspar indicate that nepheline has been replaced by K-feldspar as a result of metasomatic alterations. It has been observed that titanium bearing garnets enriched in andradite $\left(\mathrm{Ca}_{3} \mathrm{Fe}_{2}^{3+} \mathrm{Si}_{3} \mathrm{O}_{12}\right)$, schorlomite $\left(\mathrm{Ca}_{3} \mathrm{Ti}_{2}\left[\mathrm{Al}>\mathrm{Fe}_{2}^{3+}\right]_{2} \mathrm{SiO}_{12}\right)$ or melanite $\left(\mathrm{Ca}_{3} \mathrm{Ti}_{2}\left[\mathrm{Al}<\mathrm{Fe}_{2}^{3+}\right]_{2} \mathrm{SiO}_{12}\right)$ molecules principally occur in a wide variety of alkaline igneous rocks and related types including syenites (Ramaswamy 1986), nepheline syenites (Gomes 1969), ijolitemelteigites (Larsen 1942), carbonatites (Howie and Woolley 1968; Huggins et al 1977; Ramaswamy 1986). Ti-rich garnets can form over a wide range of temperatures and pressures and particularly, in alkaline rocks, they appear to reflect complex late stage metasomatic reactions of earlier-formed mafic minerals with late-stage fluids (Dingwell and Brearley 1985; Gwalani et al 2000). This idea is consistent with petrographic study of the garnet bearing assemblages from the area, which indicate that Ti-rich garnets formed from clinopyroxene and biotite, perhaps driven by metasomatic alterations. These petrographic features distinctly point towards the effects of alkali metasomatism which influenced the evolution of the SamchampiSamteran alkaline complex in a fluid-enriched milieu.

\subsection{Role of liquid immiscibility}

Anomalous enrichment of $\mathrm{P}_{2} \mathrm{O}_{5}$ in alkaline ultramafic (alkali pyroxenite) and alkaline mafic (ijolite- melteigite, alkali gabbro) rocks from SamchampiSamteran alkaline complex suggests differential dispersal of high charge density (HCD) cations like $\mathrm{P}^{5+}$ between felsic and mafic units. High charge density (HCD) cations like $\mathrm{P}^{5+}$ are incompatible with respect to the minerals formed during the early stage of fractional crystallization and are normally concentrated in late formed rocks. But, enrichment of $\mathrm{P}_{2} \mathrm{O}_{5}$ in mafic-ultramafic members of Samchampi-Samteran alkaline complex negates the idea of fractional crystallization and strongly favours liquid immiscibility as a dominant petrogenetic process for the formation of Samchampi-Samteran rocks (Philpotts 1982; Eby 1979; Subrahmanyam and Leelanandam 1989). Further, it is well documented that apatite is an effective concentrator of P (Nagasawa 1970) and occurrence of apatite in alkaline mafic-ultramafic rocks indicates significant apatite fractionation from a $\mathrm{P}_{2} \mathrm{O}_{5}$ rich melt. This apatite fractionation is favoured by a volatile/fluid enriched milieu. The process of liquid-liquid unmixing has been proposed as a suitable mechanism for producing contemporaneous liquids of felsic and mafic compositions in a variety of alkaline igneous complexes from India and other parts of the world (Markl 2001; Rajesh 2003; Halama et al 2005; Doroshkevich et al 2010). It has been documented by many researchers (Eby 1979; Philpotts 1982; Markl 2001; Rajesh 2003) that if liquid immiscibility is to produce magmatic bodies of contrasting compositions, then the parental magma has to first undergo fractional crystallization so that during immiscibility the conjugate liquids are free to split into two fractions and form felsic and mafic units. In the Greig diagram (figure 15) the SamchampiSamteran samples plot close to the two liquid field suggesting that the parental magma (possibly charged with $\mathrm{CO}_{2}, \mathrm{H}_{2} \mathrm{O}$ and $\mathrm{P}_{2} \mathrm{O}_{5}$ ) suffered pronounced liquid immiscibility. It has been suggested by Minarik (1998) that carbonate melt has a higher melt-solid interfacial energy than does the coexisting silicate melt. This behaviour prevents the carbonate melt from migrating independently from the silicate melt. Thus, for the SamchampiSamteran alkaline complex, the parent melt must have immiscibly split into a silicate liquid fraction and $\mathrm{H}_{2} \mathrm{O}-\mathrm{CO}_{2}$ rich fraction. The immiscible $\mathrm{H}_{2} \mathrm{O}-\mathrm{CO}_{2}$ rich liquid produced the carbonatite member of the complex. Field observations record lack of chilled facies and marked presence of a sharp contact between the alkaline felsic syenite member and alkaline mafic ijolite-melteigite member of the complex. Such field evidences serve as key factors supporting the role of liquid immiscibility for many alkaline-carbonatite complexes (Subrahmanyam and Leelanandam 1989). 


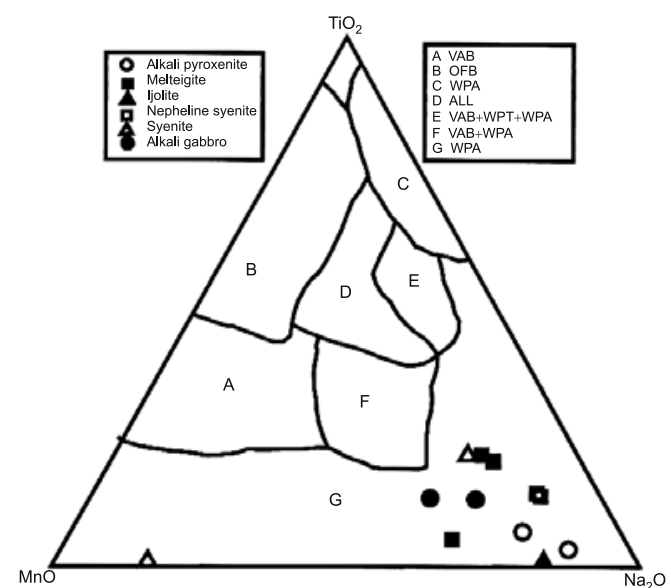

(a)

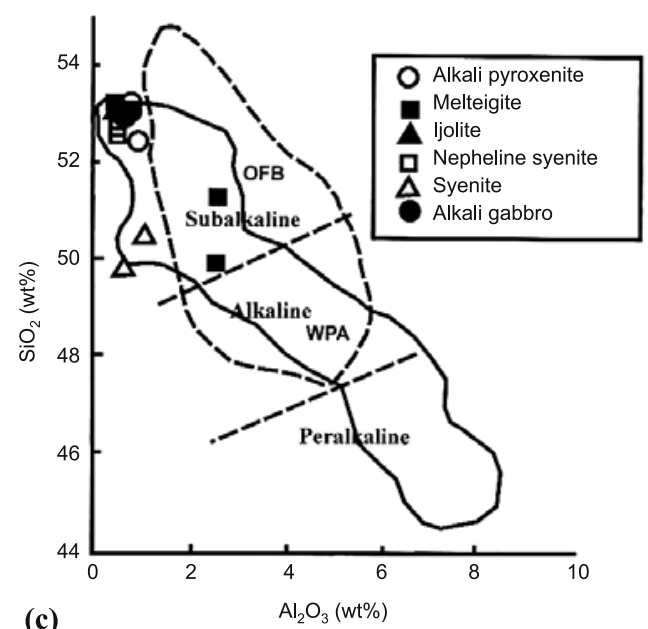

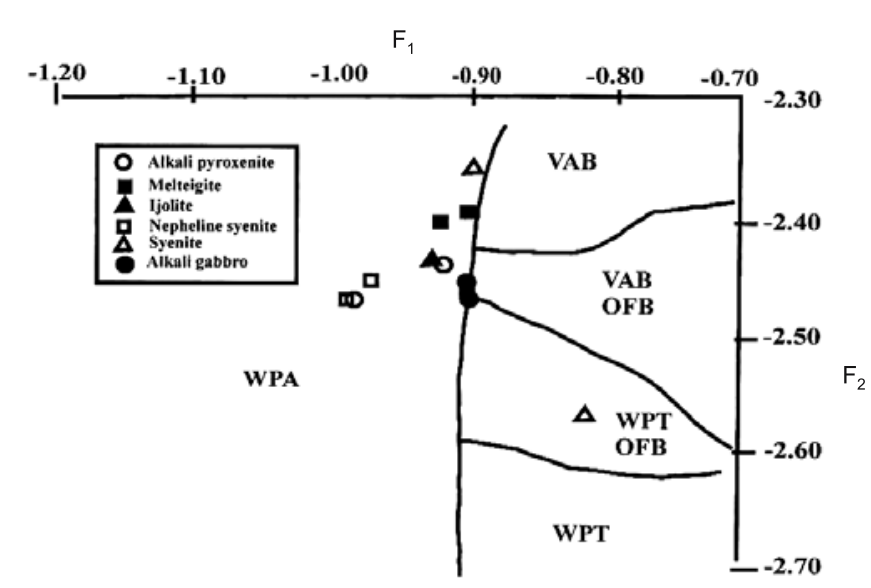

(b)

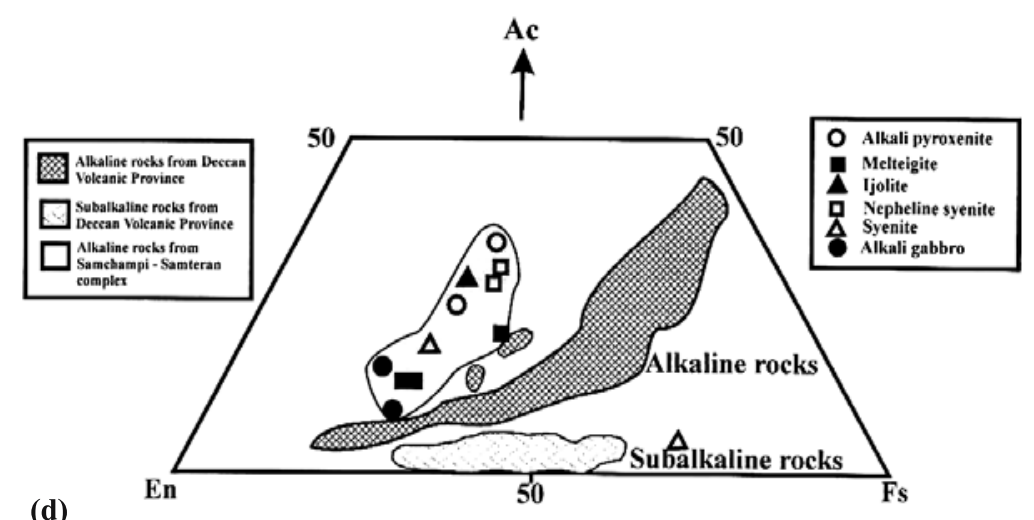

(d)

Figure 16. (a) Projection of clinopyroxene compositions of different lithotypes from Samchampi-Samteran complex in $\mathrm{TiO}_{2}-\mathrm{MnO}-\mathrm{Na}_{2} \mathrm{O}$ triangular diagram (Nisbet and Pearce 1977). VAB = Volcanic Arc Basalts, OFB = Ocean Floor Basalts, WPT $=$ Within Plate Tholeiite and $\mathrm{WPA}=$ Within Plate Alkalic basalt. (b) Plot of discriminant functions, $\mathrm{F}_{1}$ $\left(\mathrm{F}_{1}=-0.012 \times \mathrm{SiO}_{2}-0.0807 \times \mathrm{TiO}_{2}+0.0026 \times \mathrm{Al}_{2} \mathrm{O}_{3}-0.0012 \times \mathrm{FeO}^{*}-0.0026 \times \mathrm{MnO}+0.0087 \times \mathrm{MgO}-0.0128 \times \mathrm{CaO}-0.0419 \times\right.$ $\mathrm{Na}_{2} \mathrm{O}$ and $\mathrm{F}_{2}\left(\mathrm{~F}_{2}=-0.0469 \times \mathrm{SiO}_{2}-0.0818 \times \mathrm{TiO}_{2}-0.0212 \times \mathrm{Al}_{2} \mathrm{O}_{3}-0.0041 \times \mathrm{FeO}^{*}-0.1435 \times \mathrm{MnO}-0.0029 \times \mathrm{MgO}+0.0085 \times\right.$ $\mathrm{CaO}+0.0160 \times \mathrm{Na}_{2} \mathrm{O}$ ), for pyroxene analyses from Samchampi-Samteran complex (Nisbet and Pearce 1977). VAB = Volcanic Arc Basalts, OFB = Ocean Floor Basalts, WPT = Within Plate Tholeiite and WPA = Within Plate Alkalic basalt. (c) Plot of clinopyroxene compositions from Samchampi-Samteran complex in $\mathrm{SiO}_{2}$ (wt\%) vs. $\mathrm{Al}_{2} \mathrm{O}_{3}$ (wt\%) diagram (Nisbet and Pearce 1977). OFB = Ocean-Floor Basalts and WPA = Within Plate Alkalic basalt. (d) Projection of clinopyroxene compositions of different lithotypes from Samchampi-Samteran complex in terms of En-Ac-Fs components. Clinopyroxene compositions from alkaline and tholeiitic rocks of Deccan volcanic province are also shown in the diagram (Rock et al 1994; Kumar 1996).

\subsection{Evaluation of tectonic setting}

In the present study, mineral chemical parameter obtained from electron probe micro-analyses have been considered as effective tool for fingerprinting the magma type and delineating the tectonic environment of the investigated SamchampiSamteran ultramafic-mafic-alkaline-carbonatite complex. For evaluation of tectonic setting of parental magma-types, compositions of clinopyroxene were used. When the relevant compositions are projected into $\mathrm{TiO}_{2}-\mathrm{Na}_{2} \mathrm{O}-\mathrm{MnO}$ triangular diagram (Nisbet and Pearce 1977) (figure 16a), the data-plots are distinctly concentrated in the field of Within Plate Alkalic Basalts (WPA), suggesting an intraplate, anorogenic setting and a $\mathrm{Na}_{2} \mathrm{O}$ enriched alkaline character for the parent magma. The plot of clinopyroxene compositions in discriminant function diagram $\left(\mathrm{F}_{1}\right.$ vs. $\left.\mathrm{F}_{2}\right)$ (Nisbet and Pearce 1977) (figure 16b) shows that the investigated clinopyroxene has a within plate alkaline affinity. Similar within plate alkaline affinity has also been envisaged in terms of $\mathrm{SiO}_{2}$ vs. $\mathrm{Al}_{2} \mathrm{O}_{3}$ diagram (figure 16c) of clinopyroxene. Projection of clinopyroxene compositions in EnAc-Fs diagram maintains close affinity for those 
of alkaline rocks from Deccan Volcanic Province (figure 9d) (Rock et al 1994; Kumar 1996). Thus, tectonic discrimination diagrams involving clinopyroxene chemistry consistently point out a within plate alkaline affinity for the parental magma.

\subsection{Regional implications}

During the Late Jurassic-Early Cretaceous, NE India was marked by domal uparching of the Shillong Plateau accompanied by the development of $\mathrm{E}-\mathrm{W}$ trending Brahmaputra and Dauki fault systems (Gupta and Sen 1988). The Dauki fault system broke the Shillong Plateau along its southern margin and acted as a fissure for the eruption of Sylhet Traps. A concurrent feature associated with the uparching of the crust probably led to the development of $\mathrm{N}-\mathrm{S}$ and NE-SW trending lineaments and fracture zones (Gupta and Sen 1988). During the Upper Cretaceous, intrusive magmatic activities followed along these lineaments resulting in the emplacements of isolated bodies of ultramafic-mafic-alkalinecarbonatite complex. These complexes cover parts of the Shillong Plateau and Mikir Hills under a tensional regime, suggesting a deep-seated fault control and NE-SW uparching of the crust (Nandy 2001). Thus, both the phenomena of eruption of Sylhet Traps and the emplacement of differentiated alkaline complex may be related to crustal uparching and lithospheric thinning followed by fracturing and rifting. This can be attributed to the impact of an incubating mantle plume on the lithosphere. As a consequence to this effect, the crustal extension provided the passage for widespread extrusive igneous activity manifested in terms of RajmahalSylhet volcanics in eastern and northeastern India, bearing the earliest signature of hotspot magmatism in the Indian Ocean (Datta and Mitra 1984).

\section{Acknowledgements}

The authors convey their gratitude to Dr M Angamuthu, IAS, Deputy Commissioner, Karbi-Anglong and Mr P Singner, Executive Member of Parliament, Karbi-Anglong for providing their kind support during the field sessions. The authors are immensely grateful to Rev. Fr. Thankachen Joseph and Rev. Fr. Vinod of 'Jirsong Asong' for their kind co-operation during the field work. We thankfully acknowledge Mr Har Singh Kro and Mr Ken Singh, without whose help field work would not have been possible. The authors are deeply indebted to the Head, Department of Geology, University of Calcutta for proving all laboratory facilities. The authors are grateful to UGC-Major Research Project authority
[Project no: F. No. 34-51 \2008 (SR)] for providing financial assistance. Sincere thanks are due to Dr Shyamal Sengupta of the Central Petrological Laboratory, Geological Survey of India, Kolkata for his encouragement and fruitful suggestions. Two anonymous referees provided very critical and educative comments to upgrade the quality of the paper. Thanks are also due to Prof. Talat Ahmad for valuable comments and fruitful suggestions.

\section{References}

Acharya S K, Mitra N D and Nandy D R 1986 Regional geology and tectonic setting of northeast India and adjoining region; Geol. Surv. India Memoir 119 6-12.

Ai Y 1994 A revision of the garnet-clinopyroxene $\mathrm{Fe}^{2+}-\mathrm{Mg}$ exchange geothermometer; Contrib. Mineral. Petrol. 115 467-473.

Ashchepkov I V 2009 Universe clinopyroxene barometerrecalibrations on the results of the orthopyroxene thermobarometry and experimental results and applications to clinopyroxene geotherms; Abs. EGU General Assembly 11.

Bailey D K 1995 Carbonate magma; J. Geol. Soc. London $150637-651$.

Baksi A K 1995 Petrogenesis and timing of volcanism in the Rajmahal flood basalt province, Northeastern India; Chem. Geol. 121 73-90.

Basu A R, Renne P R, Das Gupta D K, Teichman F and Poreda R J 1993 Early and late alkali igneous pulses and a high ${ }^{3} \mathrm{He}$ plume origin for the Deccan flood basalts; Science 261 902-906.

Bell K 2001 Carbonatites: Relationships to mantle plume activity; In: Mantle Plumes: Their Identification through time; (eds) Erust R E and Buchan K L, Geol. Soc. Am. Spec. Paper 353 267-290.

Bell K 2002 Role of superplume in the Earth System; Tokyo International Workshop Abstract, 365-368.

Bell K, Kjarsgaard B A and Simonetti A 1998 Carbonatite into the Twenty-First Century; J. Petrol. 39 1839-1845.

Best M G 2003 Igneous and metamorphic petrology (Turin: Blackwell Publishing) 729 p.

Bindi L, Cellai D, Melluso L, Conticelli S, Morra V and Menchetti S 1999 Crystal chemistry of clinopyroxene from alkaline undersaturated rocks of the Monte Vulture Volcano, Italy; Lithos 46 259-274.

Chakhmouradian A R and McCammon C A 2005 Schorlomite: A discussion of the crystal chemistry, formula and inter-species boundaries; Physics and Chemistry of Minerals 32(4) 277-289.

Coffin M F, Pringle M G, Duncan R A, Gladezenko T P, Storey M, Millar R D and Gahagan L A 2002 Kerguelen hotspot magma output since 130 Ma; J. Petrol. 43 1121-1139.

Czamanske G K and Wones D R 1973 Oxidation during magmatic differentiation, Finnmarka complex, Oslo area, Norway, 2. The mafic silicates; J. Petrol. 14 349-380.

Das J D, Saraf A K and Jain A K 1995 Fault tectonics of the Shillong plateau and adjoining regions, north-east India using remote sensing data; Int. J. Remote Sensing 16(9) 1633-1646.

Datta N R and Mitra N D 1984 Gondwana geology of the Indian Plate - its history of fragmentation and dispersion; Geol. Surv. Japan Report 263 1-25.

Dawson J B, Smith J V and Steele I M 1995 Petrology and Mineral Chemistry of Plutonic Igneous Xenoliths 
from the Carbonatite Volcano, Oldoinyo Lengai, Tanzania; J. Petrol. 36(3) 797-826.

Deer W A, Howei R A and Zussman J 1979 An introduction to the rock forming minerals; The English Language Book Society: London 528p.

Deer W A, Howei R A and Zussman J 1982 Rock-forming minerals; Orthosilicates $2^{\text {nd }}$ edn, (New York: Longman) vol. $1 \mathrm{~A}$.

Deer W A, Howei R A and Zussman J 1992 An introduction to rock forming minerals; Prentice Hall Harrow England Person Education Limited, pp. 696.

Desikachar S V 1974 A review of the tectonic and geological history of eastern India in terms of Plate tectonic theory; J. Geol. Soc. India 15 137-149.

Dingwell D B and Brearley M 1985 Mineral chemistry of igneous melanite garnets from analcite-bearing volcanic rocks, Alberta, Canada; Contrib. Mineral. Petrol. 90 $29-35$.

Dobosi G 1987 Chemistry of clinopyroxene from the Lower Cretaceous alkaline volcanic rocks of Mecsek Mountains, South Hungary; Neus Jb Mineral Abh 156 281-301.

Dorais M J, Whitney J A and Stormer J C 1991 Mineralogical constraints on the petrogenesis of trachytic inclusions, Carpenter ridge tuff, Central San Juan volcanic field, Colorado; Contrib. Mineral. Petrol. 107 219-230.

Doroshkevich A G, Ripp G S and Moore K R 2010 Genesis of Khatula alkaline-basic Ba-Sr carbonatite complex (West Transbaikala, Russia); Mineral. Petrol. 98 245-268.

Eby G N 1979 Mount Jonhnson, Quebec - An example of silicate liquid immiscibility; Geology 7 491-494.

Ellis D J and Green D H 1979 An experimental study of the effect of $\mathrm{Ca}$ upon garnet-clinopyroxene $\mathrm{Fe}-\mathrm{Mg}$ exchange equilibria; Contrib. Mineral. Petrol. 71 13-22.

Evans P 1964 The tectonic patterns in northeastern India; Indian J. Earth Sci. 107 103-107.

Frolov A A 1973 Structural position of massifs of ultrabasicalkalic rocks and carbonatites; Geotectonics 3 27-32.

Gittins J 1989 The origin and evolution of carbonatite magma; In: Carbonatite: Genesis and Evolution (ed.) K Bell (London: Unwin Hyman) 580-600.

Gomes C B 1969 Electron microprobe analyses of zoned melanites; Am. Mineral. 54 1654-1661.

Gupta R P and Sen A K 1988 Imprints of Ninety-East Ridge in the Shillong Plateau, Indian Shield; Tectonophys. $\mathbf{1 5 4}$ 335-341.

Gwalani L G, Rock N M S, Ramaswamy R, Griffin B J and Mulai B P 2000 Complexly zoned Ti-rich melanite-schorlomite garnets from Ambadungar carbonatite-alkalic complex, Deccan Igneous Province, Gujarat State, Western India; J. Asian Earth Sci. 18 163-176.

Halama R, Vennemann T, Siebel W and Markl G 2005 The Gronnedal-Ika carbonite-syenite complex, South Greenland: Carbonatite Formation by Liquid Immiscibility; J. Petrol. 46(1) 191-217.

Hamilton D L and MacKenzie W S 1965 Phase equilibrium in the system $\mathrm{NaAlSiO}_{4}-\mathrm{KAlSiO}_{4}-\mathrm{SiO}_{2}-\mathrm{H}_{2} \mathrm{O} ;$ Mineral. Mag. 34 214-231.

Haselton H T Jr, Hovis G L, Hemingway B S and Robie R A 1983 Calorimetric investigation of the excess entropy of mixing in analbite-sanidine solid solution: Lack of evidence for $\mathrm{Na}$, $\mathrm{K}$ short-range order and implications for two feldspar thermometry; Am. Mineral. 68 398-413.

Heaman L M, Srivastava R K and Sinha A K 2002 A precise $\mathrm{U}-\mathrm{Pb}$ zircon/baddelejite age for the Jasra igneous complex, Karbi-Anglong district, Assam, NE India; Curr. Sci. 82 744-748.
Howie R A and Woolley A 1968 The role of titanium and and the effect of $\mathrm{TiO}_{2}$ on the cell-size, refractive index and specific gravity in the andradite-melanite-schorlomite series; Mineral. Mag. 36 775-790.

Huggins F E, Virgo D and Huckenholz H G 1977 Titanium containing silicate garnets. II. The crystal chemistry of melanites and schorlomites; Am. Mineral. 62 646-655.

Hyndaman D W 1985 Petrology of igneous and metamorphic rocks; McGraw-Hill Book Company, 786p.

Kent R W, Pringle M S, Muller R D, Saunders A W and Ghosh N C $2002{ }^{40} \mathrm{Ar} /{ }^{39} \mathrm{Ar}$ geochronology of the Rajmahal basalts, India, and their relationship to the Kerguelen Plateau; J. Petrol. 43 1141-1153.

Krogh E J 1988 The garnet-clinopyroxene Fe-Mg geothermometer: A reinterpretation of existing experimental data; Contrib. Mineral. Petrol. 99 44-48.

Kumar D, Mamallan R, Saravanan B, Jain S K and Krishnamurthy P 1989 Petrology and geochemistry of the Samchampi alkaline carbonatite complex, Mikir Hills, Assam, India; Exploration and Research of Atomic Minerals 2 183-199.

Kumar D, Mamallan R and Diwedy K K 1996 Carbonatite magamatism in northeast India; Journal of Southeast Asian Earth Sciences 13 145-158.

Kumar S 1996 Chemistry of clinopyroxene from subalkaline and alkaline rocks of Phenai Mata Igneous Complex, Baroda District, Gujarat, Western India; J. Geol. Soc. India 48 547-558.

Larsen E S 1942 Alkaline rocks of Iron Hill, Gunnison County, Colorado; US Geological Survey Professional Paper 197-A.

Le Bas M J 1987 Nephelinite and carbonatites; In: Alkaline igneous rocks; (eds) J G Fitton and B G J Upton, Geol. Soc. Spec. Publ. 30 58-83.

Le Bas M J 1989 Diversification of carbonatite; In: Carbonatite: Genesis and Evolution (ed.) K Bell, (London: Unwin Hyman) 428-447.

Lindsley D H 1983 Pyroxene thermometry; Am. Mineral. 68 477-493.

Macdonald G A and Katsura T 1964 Chemical composition of Hawaiian lavas; J. Petrol. 5 82-113.

Madhavan V and Khuurram Md. Z A K 1989 The alkaline gneisses of Khariar, Kalahandi district, Orissa; In: Alkaline rocks (ed.) C Leelanandam, Geol. Soc. India Memoir 15 265-289.

Mahoney J J, MacDougall J D, Lugmair G W and Gopalan K 1983 Kerguelen hotspot source for the Rajmahal Traps and Ninetyeast Ridge?; Nature 303 385-389.

Mamallan R, Kumar D and Bajpai R K 1994 Jasra ultramafic-mafic-alkaline complex: A new find in the Shillong Plateau, northeastern India; Curr. Sci. 66 64-65.

Minarik W G 1998 Complications to carbonate melt mobility due to presence of an immiscible silicate melt; J. Petrol. 39 1965-1973.

Markl G 2001 A new type of silicate liquid immiscibilty in peralkaline nepheline syenite (lujavrites) of Llimaussaq complex, South Greenland; Contrib. Mineral. Petrol. 141 $458-472$.

Mazumder S K 1986 The Precambrian framework of part of the Khasi Hills, Meghalaya; Geol. Sur. India Record 117(2) $1-58$

Melluso L, Srivastava R K, Guarino V, Zanetti A and Sinha A K 2010 Mineral chemistry and petrogenetic evolution of the ultramafic-alkaline-carbonatitic complex of Sung Valley, Northeastern India; Canadian Mineralogists 48 205-229.

Middlemost E A K 1985 Magmas and magmatic rocks; (New York: Longman) 222p. 
Mori T and Green D E 1978 Laboratory duplication of phase equilibria observed in natural garnet lherzolites; J. Geol. 86 83-97.

Morimoto N 1989 Nomenclature of Pyroxenes; Canadian Mineralogist 27 143-156.

Morimoto N, Fabries J, Ferguson A K, Ginzburg I V, Ross M, Seifert F A and Zussman J 1988 Nomenclature of pyroxenes; Am. Mineral. 73 1123-1133.

Mysen B O and Heier K S 1972 Petrogenesis of eclogite in high grade metamorphic gneisses, exemplified by the Hareidland eclogite, Western Norway; Contrib. Mineral. Petrol. 36 73-94.

Nachit H, Ibhi A, Abia E H and Ben Ohoud M 2005 Discrimination between primary magmatic biotites, reequilibrated biotites and neoformed biotites; $C R G e o-$ science 337 1415-1420.

Nag S, Sengupta S K, Gaur R K and Absar A 1999 Alkaline rocks of Samchampi-Samteran, Karbi-Anglong district, Assam, India; J. Earth Syst. Sci. 108 33-48.

Nagasawa H 1970 Rare earth concentration in zircon and apatite, and their host dacite and granite; Earth Planet. Sci. Lett. 9 359-364.

Nambiar A R 1988 Petrology of the lamprophyres from East Garo Hills and West Khasi Hills District, Meghalaya; J. Geol. Soc. India 32 125-132.

Nambiar A R 2007 Early Cretaceous Lamprophyre Dykes from Nongchram Fault Zone, Meghalaya, Northeastern India; J. Geol. Soc. India 69 641-652.

Nandy D R 1980 Tectonic patterns in northeastern India; Indian J. Earth Sci. 7 103-107.

Nandy D R 2001 Geodynamics of North Eastern India and the adjoining region (Calcutta: ACB Publication) $120 \mathrm{p}$.

Nisbet E G and Pearce J A 1977 Clinopyroxene composition in mafic lavas from different tectonic settings; Contrib. Mineral. Petrol. 63 149-160.

Pattison D R M and Newton R C 1989 Reversed experimental calibration of the gamet-clinopyroxene $\mathrm{Fe}-\mathrm{Mg}$ exchange thermometer; Contrib. Mineral. Petrol. 101 $87-103$.

Philpotts A R 1982 Composition of immiscible liquids in volcanic rocks; Contrib. Mineral. Petrol. 80 201-218.

Raheim A and Green D H 1974 Experimental determination of the temperature and pressure dependence of the Fe-Mg partition coefficient for coexisting garnet and clinopyroxene; Contrib. Mineral. Petrol. 48 179-203.

Rajesh H M 2003 Outcrop-scale silicate liquid immiscibility from an alkali syenite (A-type granitoid)-pyroxenite association near Puttetti, Trivandrum Block, South India; Contrib. Mineral. Petrol. 145 612-627.

Ramaswamy R 1986 Titanium-bearing garnets from alkaline rocks of carbonatite complex, Tiruppattur, Tamil Nadu; Curr. Sci. 55 1026-1029.

Ratnakar J and Leelanandam C 1989 Petrology of the alkaline plutons from the eastern and southern peninsular India; Geol. Soc. India Memoir 15 145-176.

Ray J S, Ramesh R and Pande K 1999 Carbon isotopes in Kerguelen plume-derived carbonatites: Evidence for recycled inorganic carbon; Earth Planet. Sci. Lett. 170 $205-214$

Ray J S and Pande K $2001{ }^{40} \mathrm{Ar}-{ }^{39} \mathrm{Ar}$ age of carbonatitealkaline magmatism in Sung Valley, Meghalaya, India; J. Earth Syst. Sci. 110 185-190.

Renne R, Glen J M, Milner S C and Duncan A R 1996 Age of Etendeka flood volcanism and associated intrusions; Geology 24 659-662.

Rock N M S 1990 The International Mineralogical Association (IMA/CNMMN) pyroxene nomenclature scheme: Computerization and its consequences; Mineral. Petrol. 43 99-119.

Rock N M S, Gwalani L G and Griffin B J 1994 Alkaline rocks and carbonatites of Amba Dongar and adjacent areas, Deccan Alkaline Province, Gujarat, India. 2: Complexly zoned clinopyroxene phenocrysts; Mineral. Petrol. 51 113-136.

Sengupta P K, Dasgupta S, Bhattacharyya P K and Hariya Y 1989 Mixing behaviour in quaternary gamet solid solution and an extended Ellis and Green garnetclinopyroxene geothermometer; Contrib. Mineral. Petrol. $103223-227$.

Srivastava R K and Sinha A K 2004 Geochemistry of Early Cretaceous alkaline ultramafic-mafic complex from Jasra, Karbi Anglong, Shillong Plateau, Northeastern India; Gondwana Res. 7(2) 549-561.

Srivastava R K, Heaman L M, Sinha A K and Shihua S 2005 Emplacement age and isotope geochemistry of Sung Valley Alkaline-Carbonatite complex, Shillong Plateau, Northeastern India: Implications for primary carbonate melt and genesis of the associated silicate rocks; Lithos $8133-54$.

Srivastava R K and Sinha A K 2007 Nd and Sr isotope systematics and geochemistry of plume related early Cretaceous alkaline-mafic-ultramafic igneous complex from Jasra, Shillong Plateau, Northeastern India; Geol. Soc. Am. Spec. Paper 430 815-830.

Streckeisen A 1976 To each plutonic rock its proper name; Earth Sci. Rev. 12 1-33.

Subrahmanyam N P and Leelanandam C 1989 Differentiation due to probable initial immiscibility in the Musala Pluton of the Mundwara Alkali Igneous Complex, Rajasthan, India: In: Alkaline rocks (ed.) C Leelanandam, Geol. Soc. India Memoir 15 25-46.

Thornton C P and Tuttle O F 1960 Chemistry of igneous rocks: pt. 1, differentiation index; Am. J. Sci. $\mathbf{2 5 8}$ 664-684.

Veena K, Pandey B K, Krishnamurthy P and Gupta J N $1998 \mathrm{~Pb}, \mathrm{Sr}$ and $\mathrm{Nd}$ isotope systematics of Sung Valley, Meghalaya, Northeast India: Implications for contemporary plume-related mantle source characteristics; J. Petrol. 39 1975-1984.

Woolley A R 1989 The spatial and temporal distribution of carbonatite; In: Carbonatite genesis and evolution (ed.) K Bell (London: Unwin Hyman) 15-37.

Zen E 1988 Phase relations of peraluminous granitic rocks and their petrogenetic implications; Earth Planet. Sci. Ann. Rev. 16 21-51. 